Historic, Archive Document

Do not assume content reflects current scientific knowledge, policies, or practices. 



\section{$a^{5011}$ - A42 Reence \\ Regional Demand and Supply Projections for Outdoor Recreation}

United States

Agriculture

Forest Service

Rocky Mountain

Forest and Range

Experiment Station
Donald B. K. English, Carter J. Betz, J. Mark Young, John C. Bergstrom, and $\mathrm{H}$. Ken Cordell

Fort Collins

Colorado 80526

General Technical

Report RM-230

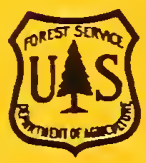

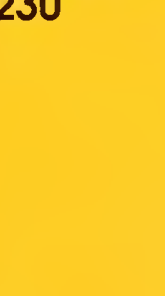




\section{Acknowledgments}

The authors appreciate the instructive comments and suggestions from the following reviewers-Robert W. Douglass (Ohio State University), Linda Langner (USDA Forest Service), Richard W. Paterson (Tennessee Valley Authority), and Chrystos D. Siderelis (North CarolinaState University). Appreciation is extended to Shela Mou for assistance with the manuscript preparation. Any remaining errors or omissions are the responsibility of the authors.

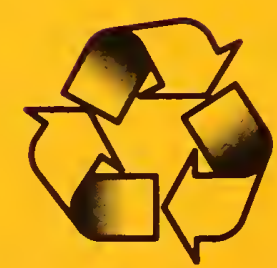

This publication was printed on recycled paper. 


\title{
Regional Demand and Supply Projections for Outdoor Recreation
}

\author{
Donald B. K. English, Research Social Scientist ${ }^{1}$ \\ Carter J. Betz, Outdoor Recreation Planner' \\ J. Mark Young, Outdoor Recreation Planner ${ }^{1}$ \\ John C. Bergstrom, Associate Professor, University of Georgia \\ H. Ken Cordell, Research Scientist ${ }^{1}$
}




\section{Contents}

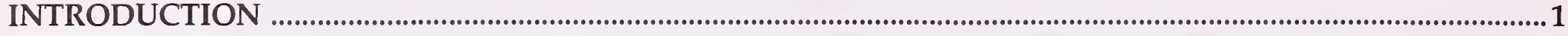

STUDY OBJECTIVES ............................................................................................................................................... 2

THEORETICAL BACKGROUND ........................................................................................................................

DEMAND AND SUPPLY OF TRIPS ....................................................................................................................2

RECREATION OPPORTUNITY INDEX ......................................................................................................................

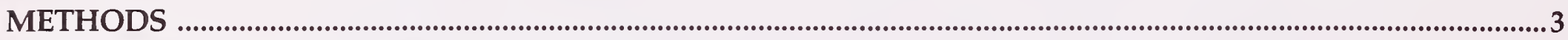

RECREATION TRIP DEMAND AND SUPPLY ............................................................................................................

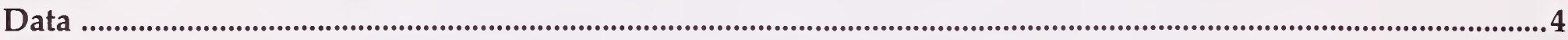

Model Specification ..................................................................................................................................................

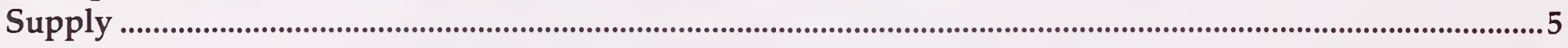

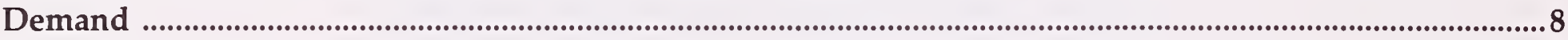

Projections ........................................................................................................................................................................11

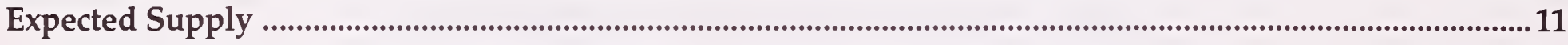

Maximum Preferred Demand .......................................................................................................................................11

Estimates of Recreation Trips ..........................................................................................................................................13

RECREATION OPPORTUNITY INDICES ...............................................................................................................

Updating Key Variables ..................................................................................................................................................15

Regional EROS Calculation ..................................................................................................................................15

Projections ..........................................................................................................................................................16

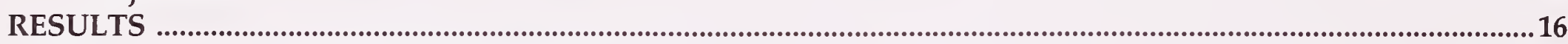

REGIONAL POPULATION COMPARISONS ……………………............................................................................16

Current Situation ...............................................................................................................................................................16

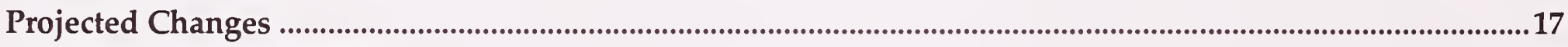

RESOURCE OPPORTUNITIES ................................................................................................................................

Key Variable Updates ........................................................................................................................................................17

Regional EROS Indices .................................................................................................................................................... 18

Substitute Recreation Opportunities .................................................................................................................................. 20

Regional EROS Projections................................................................................................................................................20

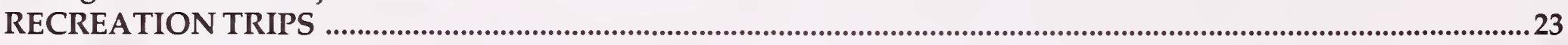

Current Consumption .............................................................................................................................................................23

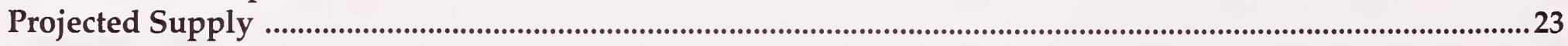

Projected Demand ................................................................................................................................................................... 28

COMPARISON OF DEMAND AND SUPPLY .........................................................................................................28

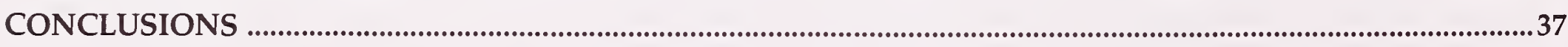

LITERATURE CITED ............................................................................................................................... 


\title{
Regional Demand and Supply Projections for Outdoor Recreation
}

\author{
Donald B. K. English, Carter J. Betz, J. Mark Young, John C. Bergstrom, and H. Ken Cordell
}

INTRODUCTION

TheForestand Rangeland Renewable ResourcesPlanning Act of 1974 (RPA), requires the USDA Forest Service to conduct an assessment of national level economic trends in renewable resources, including outdoor recreation, every 10 years. The 1989 RPA Assessment of Outdoor Recreation and Wilderness (Cordell and others 1990) provided national estimates and projections of the demand for and supply of recreation trips for 31 activities. ${ }^{2}$ National-level models, however, can obscure important regional differences in recreation

\footnotetext{
2Estimates and projections of wildlife and fish recreation were reported in Flather and Hoekstra (1989); however, this reportincludes the activity "wildlife observation."
}

preferences, travel behavior, population diversity and growth trends, demand trends, and availability of opportunities. Regional differences in recreation demands and trends also can have important implications for planning and programming at regional and subregional levels. This study, an issue analysis for the 1993 Update of the 1989 RPA Assessment, was designed to provide regionally disaggregated descriptions and projections of possible future recreation consumption and of the supply of recreation opportunities. The four regions of interest were the same as those defined for the 1989 RPA Assessment: North, Pacific Coast, Rocky Mountains and Great Plains (hereafter shortened to "Rocky Mountains"), and South (fig. 1).

\section{Forest Service Regions and Assessment Regions}

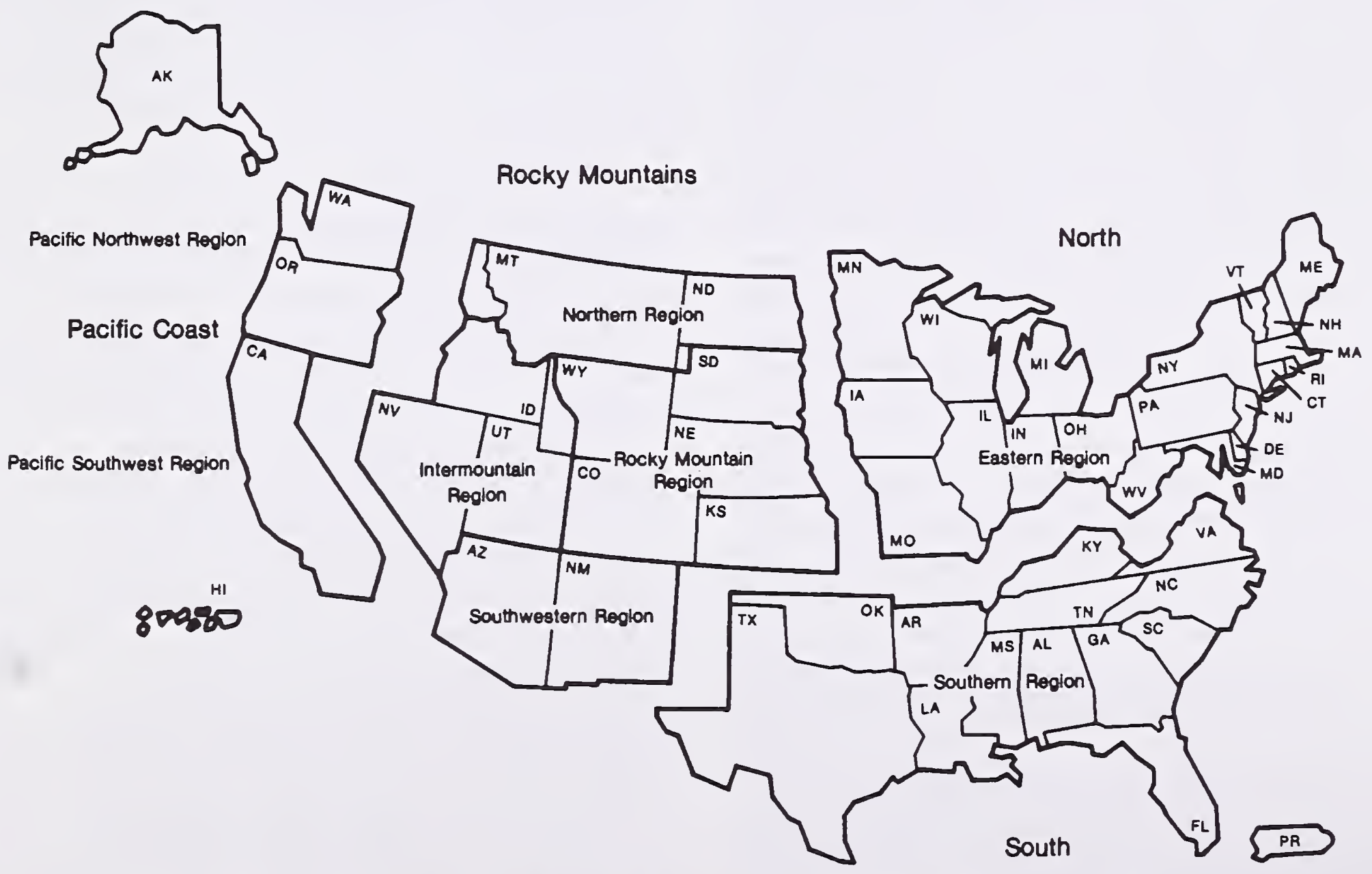

Figure 1.-Forest Service Regions and Assessment Regions. 


\section{STUDY OBJECTIVES}

The principal objectives for the recreation resource opportunity portion of this study were to update key resource data used in the 1989 RPA Assessment, and to develop region-specific recreation opportunity effectiveness ratings. These objectives update measures of the effective supply of available outdoor recreation opportunities, and reflect regional differences in resource availability.

The primary objective of the recreation activity analysis was to adapt all projection models to regional bases. Two additional recreation activities not included in the 1989 RPA Assessment — sailing and snowmobiling were modeled for the 1993 Update.

\section{THEORETICAL BACKGROUND}

\section{DEMAND AND SUPPLY OF TRIPS}

Research has shown that one appropriate conceptual model for studying recreation demand and supply is that of household trip production (Bockstael and McConnell 1981, Cordell and Bergstrom 1991). It is widely accepted that the most appropriate measurement unit for recreation demand analyses is the recreation trip (McConnell 1975, Cordell and Bergstrom 1991). Typically, trips are not traded in traditional economicmarkets. Instead, recreating households are both the producers and consumers of recreation trips. In the joint production/consumption process, households combine their time, skills, and knowledge with market inputs and existing recreation opportunities. Thus, for the household production model, both supply and demand of recreation trips are determined from the household's perspective (Becker 1965, Bockstael and McConnell 1981).

A primary objective of the Forest Service's RPA Assessment of Outdoor Recreation is to compare projected demand and supply of recreation trips. The supply side is represented by projected trends in trip production and consumption at future points in time, given expected constraints on land, water, and other resources available for recreation (Cordell and Bergstrom 1991). As resource availability changes, so will average trip prices, increasing as resources become more scarce. In this study, the projected "supply" of trips is synonymous with the projected number of trips that are expected to be produced andconsumed. These projections have been labeled the "expected supply" of trips (Cordell and Bergstrom 1991).
Future demand for recreation trips is measured by the number of trips households would take if the future trip costs remain unchanged and resource availabilities are unconstrained. This measure, termed "maximum preferred demand"" (Cordell and Bergstrom 1991), is interpreted as the number of trips households would prefer to take if trip costs remained constant into the future relative to the base year, 1987. Here, resource opportunities are assumed to grow or contract as necessary without constraint to meet changes in use and population so that trip cost and quality remain constant.

Although maximum preferred demand and expected supply both are predicted future consumption scenarios, the difference lies in the assumptions about future trip cost and recreation opportunity. Maximum preferred demand holds trip prices constant at the base year (1987) level, which, in turn, means that recreation resource availability is essentially unconstrained. Maximum preferred demand answers the question, "How many activity trips would American households demand given no change in their per trip cost?" Expected supply, in contrast, leaves trip prices unconstrained, but constrains the amount of resource based on an extension of recent past trends for 12 different recreation resource environments. For some resource environments, the trend indicates future growth; for others, it indicates decline. Expected supply answers the question, "How many activity trips would American households produce and consume if resources change at the same rate as recent past trends?"

For specified future years, projections of expected supply and maximum preferred demand are computed and compared. A "gap" or shortage occurs where projected demand for a specified year exceeds projected supply (Cordell and others 1990). Such a gap indicates that resource or cost constraints prohibit households from producing as many recreation trips as they would prefer to produce, if the relative availability of resources was unconstrained and trip costs unchanged from the base year condition. Reductions in the amount of resources available for recreation is a major factor determining costs of producing trips, primarilybecausehouseholds must travel greater distances to recreate or face reduced quality from congested sites.

Gaps are largest for those activities and regions where both recreation resource availabilities are projected to decline, thus reducing expected supply, and the effects of changes in population, income, and other household characteristics are projected to be relatively large, thus driving up maximum preferred demand. When the 
projected supply equals or exceeds the projected demand, households will produce as many trips as they prefer, and no gap results. Gaps typically are minimal to nonexistent where the projected rate of resource growth is increasing enough to keep pace with increases in population and income.

\section{RECREATION OPPORTUNITY INDEX}

The Effective Recreation Opportunity Set (EROS) index is a measure of the general availability of recreation opportunities, and can be used in models of household demand and consumption of recreation trips (English and Cordell 1993). Common measures of recreation resources, such as raw facility counts or facilities percapita, have beenshown to beinadequate(Harrington 1987). Economic concepts of recreation supply (Clawson 1984, Harrington 1987) are difficult to calculate empirically. Opportunity indices are effective measures of the joint spatial distribution of recreation opportunities and households (Fesenmaier and Leiber 1987; Kim and Fesenmaier 1990). EROS indices are based on this above research, and particularly on Harrington's (1987) 'effec-

Table 1.-Twelve types of recreation environments used as the basis for effective recreation opportunity set (EROS) indices.

\section{Category Description}

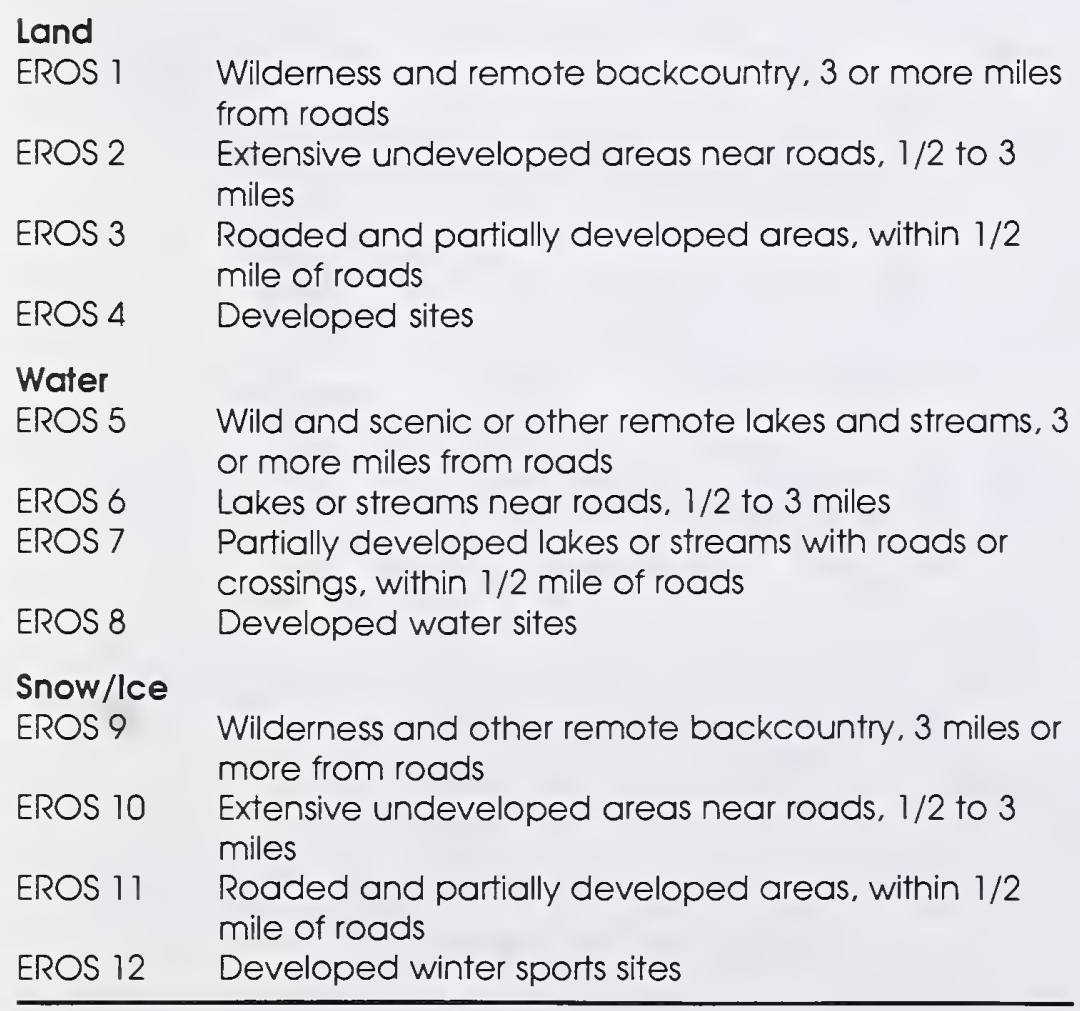

Source: Cordell and others (1990) tive price' measure, and opportunity indices. A separate EROS index was calculated for each of the 12 recreation environments identified by Cordell and others (1990) for the 1989 RPA Assessment, four each within land, water, and snow and ice resources (table 1).

The household production model shows that recreation resources are one of several inputs to trip production. Households do not "'buy"' a site; but, they do pay a cost to acquire its use. Harrington's (1987) 'effective price' measures a recreation site's availability to a household. Effective price includes entry fees, travel costs in both money and time, and congestion costs in both queuing time and experience quality. Travel costs are the primary determinant of site availability, are specific to an origin-destination pair, and are assumed to increase with distance. Sites beyond some threshold distance become too expensive to use. Threshold distances vary by recreation setting (Cordell and English 1985). More specifically, threshold distances vary by activity. For example, a household may be willing to drive 10 miles or less to go sledding but would willingly travel several hundred miles for downhill skiing. However, the EROS indices are not activity specific, instead, they correspond to more general recreation environments or settings.

Trip quality declines as the number of users at a site increases, because of congestion and queuing (Harrington 1987). Converting quality decline to an equivalent price variation allows congestion to be treated as a cost. Congestion costs depend on the total number of people at the site. Numbers of users at a site depends on the location and size of population centers and other recreation sites within the appropriate threshold distance (Fesenmaier and Leiber 1987; Kim and Fesenmaier 1990).

EROS indices include travel costs and congestion components (English and Cordell 1993). County-level resolution for population and resource data drive the calculation method. Distance separating spatial units (counties) provides proxies for mean travel distance separating the units.

\section{METHODS}

\section{RECREATION TRIP DEMAND AND SUPPLY}

The1993Updateused thesameset of aggregatedata that was used in the 1989 Assessment. The same methods used to develop national projections for the 1989 Assessment were applied to each of the four regions. The main differ- 
ence between the 1989 RPA Assessment and the 1993 RPA Update was that projections of two key independent variables - population and household income-varied by region in this report. A brief summary of the model specifications, data sources, and theoretical background used in the 1989 Assessment (and repeated for the 1993 RPA Update) is presented here. Cordell and Bergstrom (1991) provide a more complete discussion.

\section{Data}

Individual survey data from the Public Area Recreation Visitor Study (PARVS) were aggregated to multicounty trip-generating regions containing at least 90 individual responses (Cordell and Bergstrom 1989). PARVS was a cooperative research effort involving more than a dozen federal and state natural resource agencies. Recreationists were interviewed at more than 250 public recreation areasnationwide. Aggregate, rather than household, data were used, because of the RPA goal of analyzing broad, nationwide trends in outdoor recreation. About 26,000 individual responses were used to create 239 aggregate observations for the 1989 RPA Assessment modeling. For each multi-county re- gion, a single representative county was chosen (fig. 2), based on being the home county of a majority of the individual responses, or if no county had a majority, proximity to the region's geographic center. Recreation behavior was assumed to be homogeneous within a region; so, trips from all cases in the multi-county region were used to construct the dependent variable. For the 1993 Update, each representative county was assigned to the appropriate region.

For each activity, the number of trips per capita for all respondents within a region was multiplied by the representative county's population over age 11, to estimate the dependent variable, total annual activity $k$ trips generated by the representative county. Projections of future demand and supply were calculated by inserting expected future values for independent variables into the estimated regression model equations and solving. For the 1993 RPA Update, regional estimates of population and household income provided by the Bureau of Economic Analysis (U.S. Department of Commerce 1991) were inserted in place of national estimates derived from U.S. Census Bureau data. This region-specific information assured that variation would exist between the regional projections of recreation demand and supply.

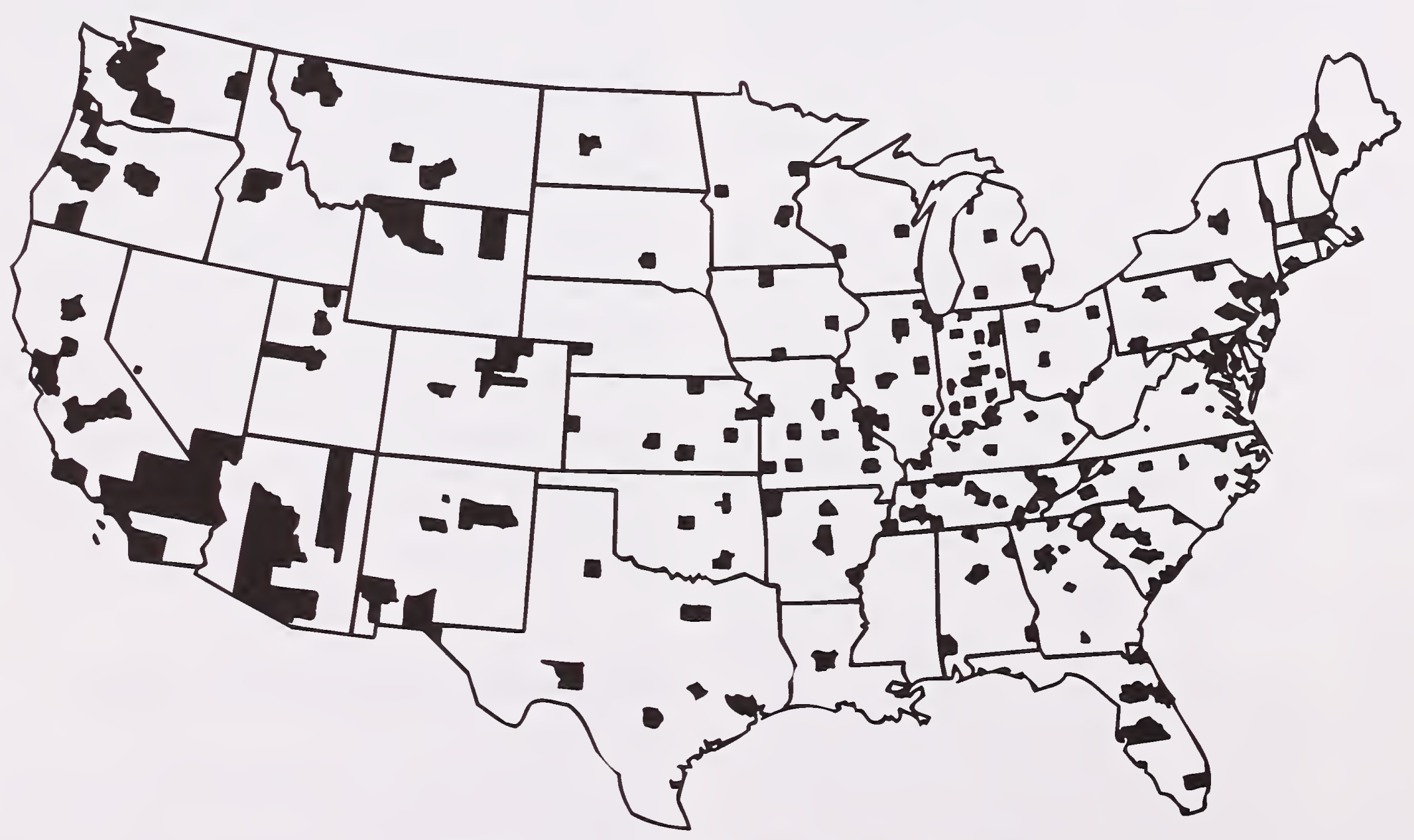

Figure 2.-Representative counties used in RPA Assessment modeling. 


\section{Model Specification}

\section{Supply}

The supply of outdoor recreation trips is defined as the number of trips that households would actually produce/consume under household production theory. The general model of recreation consumption is:

ATRIPS $=\mathrm{f}\left(\mathrm{SO}, \mathrm{Z}, \mathrm{S}, \mathrm{RO}_{\mathrm{k}^{\prime}} \mathrm{H}\right)$

where

\begin{tabular}{|c|c|}
\hline TRIPS & $\begin{array}{l}=\text { annual number of trips for activity } \mathrm{k} \\
\text { consumed by a community, }\end{array}$ \\
\hline $\mathrm{O}$ & $\begin{array}{l}\text { substitute recreation opportunities } \\
\text { available to a community }\end{array}$ \\
\hline & $\begin{array}{l}\text { community population } 12 \text { years old and } \\
\text { older }\end{array}$ \\
\hline & suitability of sites used for activity k \\
\hline $\mathrm{O}_{\mathrm{k}}$ & $\begin{array}{l}\text { recreation opportunities available } \\
\text { to a community for activity } k\end{array}$ \\
\hline & community characteristics \\
\hline
\end{tabular}

Salient community characteristics shown in past research to be related to recreation consumption include income, age, and rural/urban residence. Measures of these variables were: percent of households with annual income of $\$ 30,000$ or more, percent of the population that was between the ages of 18 and 32 , and percent of the population living on farms. The equation estimated for the 1989 Assessment and used in the 1993 Update analyses was:

$$
\begin{aligned}
\ln \left(\text { TRIPS }_{\mathrm{ki}}\right) & =\beta_{0}+\beta_{1} \text { INC345 }_{\mathrm{i}} \\
& +\beta_{2} \text { PCT18TMD }_{\mathrm{i}}+\beta_{3} \text { CCPOP86 }_{\mathrm{i}} \\
& -\beta_{4} \text { PCTFARM }_{\mathrm{i}}-\beta_{5} \text { SUBEROS }_{\mathrm{ki}} \\
& +\beta_{6} \text { FACILITY }_{\mathrm{ki}} * \text { SUIT }_{\mathrm{ki}}
\end{aligned}
$$

where

$$
\begin{aligned}
& \text { TRIPS }_{\mathrm{ki}}=\text { natural log of annual activity } \mathrm{k} \\
& \text { SUBEROS }_{\mathrm{ki}}=\text { index of recreation opportunities }
\end{aligned}
$$

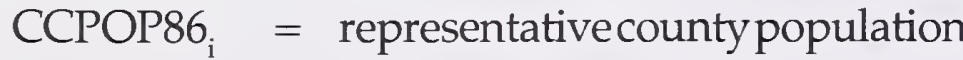

$$
\begin{aligned}
& 12 \text { years old and older, }
\end{aligned}
$$

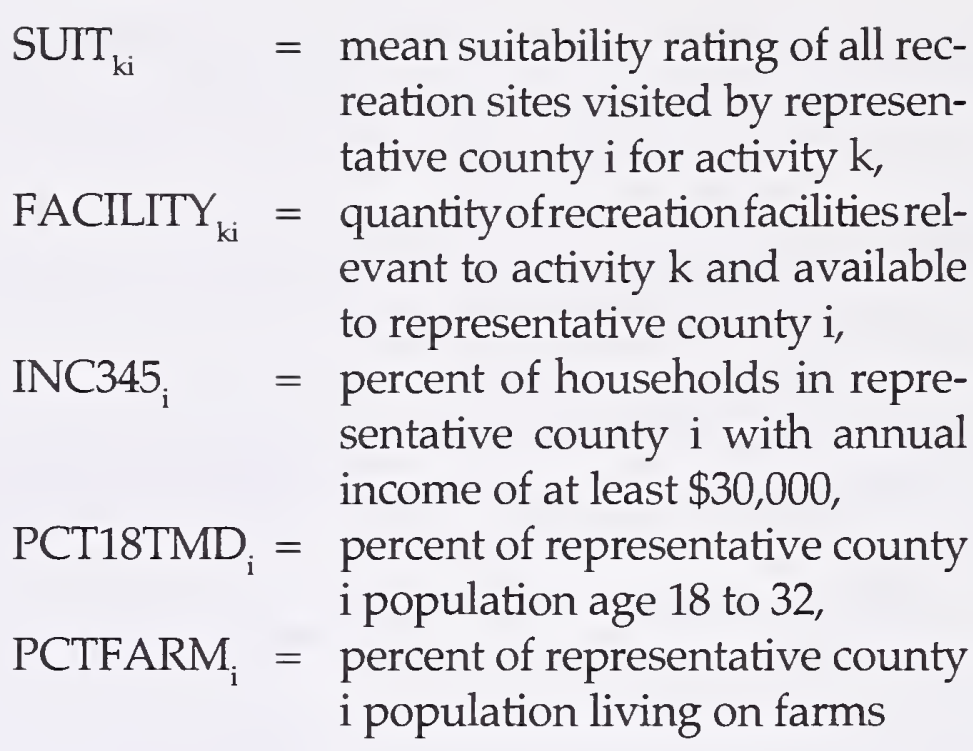

Substitute recreation opportunities (SUBEROS) and recreation resources and facilities (FACILITY) differed depending on the recreation activity being modeled; each activity model contained at least one unique facility or resource variable (table 2). Generally, resource variables were weighted by their suitability for the particular activity. A few resources, such as the number of outdoor swimming pools for outdoor pool swimming, and hourly ski lift capacity for downhill skiing, were perfectly suited to the respective activity and, therefore, were unweighted.

Each activity model was assigned a substitute recreation opportunities index variable (SUBEROS) coinciding with the environment in which the activity predominantly occurs. For example, backpacking was assigned SUBEROS1, wilderness and other remote lands, because most backpacking trips take place in wilderness and other extensive roadless areas. TheSUBEROS index is the mean of those Effective Recreation Opportunity Set (EROS) indices whose resource categories are reasonable substitutes for the environment of the target activity. EROS indices are described in detail later in this report.

Community consumption functions estimated at the national level are shown in table 3 . These are the consumption models estimated for the 1989 RPA Assessment that were used to predictexpected supply (Cordell and others 1990; Cordell and Bergstrom 1991). Model coefficients were then used in deriving regional projections of the expected supply of recreation trips for the 1993 RPA Update.

Differences between 1989 RPA Assessment and 1993 RPA Update.-For the 1993 RPA Update, equations were not re-estimated for each region. To retain consistency with the 1989 RPA results, the same intercepts and coefficients estimated in 1989 were applied 


\section{Developed camping}

Picnicking

Sightseeing

Family gatherings

Pleasure driving

Visiting historical sites

Attending events

Visiting museums

Off-road driving

Biking

Running/jogging

Walking

Cutting firewood

Collecting berries

\section{Land}

Federal road mileage converted to acres and federal and state land located within $1 / 2$ mile of a road*

Federal and state land located within $1 / 2$ mile of a road, and state forest land open to recreation*

Federal road mileage converted to acres, and federal and state land located within $1 / 2$ mile of a road"

Federal road state, local and private campgrounds*

Federal road mileage converted to acres, and federal and state land located within $1 / 2$ mile of a road

Federal road mileage converted to acres, and federal and state land located with $1 / 2$ mile of a road*

Federal road mileage converted to acres, and federal and state land located within $1 / 2$ mile of a road*

Federal road mileage (except for U.S. Army Corps of Engineers and Tennessee Valley Authority) converted to acres, National Recreational Trail mileage open to motorcycles converted to acres, and federal and state land located within $1 / 2$ mile of a road*

Federal road mileage converted to acres, and federal and state land located within $1 / 2$ mile of a road"

Federal road mileage converted to acres, federal and state land located within 1/2 mile of a road, and state forest acres open to recreation *

Federal road mileage converted to acres, federal and state land located within $1 / 2$ mile of a road, and state forest acres open to recreation *

Federal road mileage converted to acres, and federal and state land located within $1 / 2$ mile of a road*

Federal land located within $1 / 2$ mile of a road, federal and state land located within $1 / 2$ to 3 miles of a road, and acres of nonindustrial forest land open to recreation, both leased and nonleased*

Industrial and nonindustrial forest lands" 
Visiting prehistoric sites

Photography

Day hiking

Horseback riding

Nature Study

\section{Backpacking}

Primitive camping

Wildlife observation

Pool swimming

Motorized boating

Water-skiing

Rafting/tubing
Federal road mileage converted to acres, federal and state land located within $1 / 2$ mile of a road, federal and state land located within $1 / 2$ to 3 miles of a road, and rural transportation use acres*

Federal and state land located within $1 / 2$ mile of a road, and state forest acres open to recreation*

Federal and state land located within $1 / 2$ mile of a road, federal and state land located $1 / 2$ to 3 miles of a road, and federal wilderness*

Federal and state land located within $1 / 2$ to 3 miles of a road, and nonwilderness land more than 3 miles from a road

Acres of water in river/streams up to 660 feet wide, and acres of flat-water bodies

Federal and state land located within $1 / 2$ to 3 miles of a road, nonwilderness land located over 3 miles from a road, and federal wilderness acres*

Federal and state land located within $1 / 2$ mile of a road, and state forest acres open to recreation*

Federal and state land located within $1 / 2$ mile of a road, federal and state land located within $1 / 2$ to 3 miles from a road, nonwilderness land located more than 3 miles from a road, federal wilderness acres, The Nature Conservancy acres, and state fish and game land*

\section{Water}

Public and private swimming pools, state parks with some swimming facilities, and tourist accommodations

Acres of flatwater bodies and acres of federal water open to recreation

Acres of flatwater bodies and acres of federal water open to recreation*

Miles of federal wild and scenic rivers, miles of rivers designated by states as being significant for historic, cultural, scenic or recreational reasons, and miles of Bureau of Land Management recreation rivers*
Miles of National Recreational Trails open to horseback riding

Federal and state land located within $1 / 2$ mile to 3 miles of a road, nonwilderness land located over 3 miles from a road, and federal wilderness acres*

National Recreation Trail state park trail miles*

Acres of water in rivers/streams up to 660 feet wide, acres of flat-water bodies, and acres of federal water bodies open to recreation*

Number of boat ramps*

Indicator variable for presence of mountains $(\mathrm{O}=$ no mountains: $l=$ mountains) ${ }^{*}$ 
Canoeing/kayaking

Rowing/paddling, etc.

Stream/lake/ocean swimming

Sailing

Acres of flatwater bodies, and acres of water in river/streams up to 660 feet wide*

Acres of flatwater bodies, acres of water in rivers/ streams up to 660 feet wide, and acres of federal water bodies open to recreation*

Federal developed swimming areas*

Number of boat ramps*

\section{Snow and lce}

Downhill skiing

Cross-country skiing

Snowmobiling
Daily ski-lift capacity

Federal and state lands located within $1 / 2$ mile of a road, federal and state lands located within $1 / 2$ to 3 miles of a road, and acres of rural transportation use*

Federal and state lands located within $1 / 2$ mile of a road, federal and state lands located within $1 / 2$ to 3 miles of a road, and acres of rural transportation use*
Canoe rental firms and canoe outfitters*

Miles of public ocean beach* 
Land

\begin{tabular}{|c|c|c|c|c|c|c|c|c|c|c|}
\hline Developed camping & $\begin{array}{l}8.253^{*} \\
(.750)\end{array}$ & $\begin{array}{l}0.065^{*} \\
(.010)\end{array}$ & $\begin{array}{l}0.084^{*} \\
(.033)\end{array}$ & $\begin{array}{c}0.0000012^{*} \\
(1.34 \mathrm{E}-07)\end{array}$ & $\begin{array}{r}-0.060^{*} \\
(.014)\end{array}$ & - & $\begin{array}{l}0.0000047^{*} \\
(.0000014)\end{array}$ & - & 239 & 49.488 \\
\hline Picnicking & $\begin{array}{l}8.765^{*} \\
(.718)\end{array}$ & $\begin{array}{l}.051^{*} \\
(.009)\end{array}$ & $\begin{array}{l}.118 \\
(.032)\end{array}$ & $\begin{array}{c}.0000012^{*} \\
(1.30 \mathrm{E}-07)\end{array}$ & $\begin{array}{l}-.071^{*} \\
(.014)\end{array}$ & - & $\begin{array}{l}.000044^{*} \\
(.00001)\end{array}$ & - & 239 & 54.607 \\
\hline Sightseeing & $\begin{array}{r}10.885^{*} \\
(.633)\end{array}$ & $\begin{array}{l}.024^{*} \\
(.009)\end{array}$ & $\begin{array}{l}.108^{*} \\
(.027)\end{array}$ & $\begin{array}{c}.0000010^{*} \\
(1.12 \mathrm{E}-07)\end{array}$ & $\begin{array}{l}-.045^{*} \\
(.012)\end{array}$ & $\begin{array}{c}-0.189^{*} \\
(.018)\end{array}$ & $\begin{array}{l}.0000019^{*} \\
(.000001)\end{array}$ & - & 239 & 80.838 \\
\hline Family gatherings & $\begin{array}{l}8.604^{*} \\
(.777)\end{array}$ & $\begin{array}{l}.062^{*} \\
(.010)\end{array}$ & $\begin{array}{l}.087^{*} \\
(.037)\end{array}$ & $\begin{array}{c}.0000013^{*} \\
(1.41 \mathrm{E}-07)\end{array}$ & $\begin{array}{l}-.060^{*} \\
(.013)\end{array}$ & - & $\begin{array}{l}.00024^{*} \\
(.0001)\end{array}$ & - & 239 & 57.467 \\
\hline Pleasure driving & $\begin{array}{l}9.579^{*} \\
(.727)\end{array}$ & $\begin{array}{l}.061^{*} \\
(.01)\end{array}$ & $\begin{array}{l}.103^{*} \\
(.032)\end{array}$ & $\begin{array}{c}.0000012^{*} \\
(1.32 \mathrm{E}-07)\end{array}$ & $\begin{array}{l}-.058^{*} \\
(.014)\end{array}$ & - & $\begin{array}{l}.0000036^{*} \\
(.000001)\end{array}$ & - & 239 & 51.895 \\
\hline Visiting historical sites & $\begin{array}{l}8.755^{*} \\
(.663)\end{array}$ & $\begin{array}{l}.039^{*} \\
(.009)\end{array}$ & $\begin{array}{l}.135^{*} \\
(.029)\end{array}$ & $\begin{array}{l}.00000012^{*} \\
(1.18 \mathrm{E}-07)\end{array}$ & $\begin{array}{l}-.054^{*} \\
(.012)\end{array}$ & $\begin{array}{l}-.205^{*} \\
(.019)\end{array}$ & $\begin{array}{l}.0000032^{*} \\
(.000002)\end{array}$ & - & 239 & 87.398 \\
\hline Attending events & $\begin{array}{l}7.353^{*} \\
(.761)\end{array}$ & $\begin{array}{l}.068^{*} \\
(.010)\end{array}$ & $\begin{array}{l}.123^{*} \\
(.034)\end{array}$ & $\begin{array}{c}.0000012^{*} \\
(1.37 \mathrm{E}-07)\end{array}$ & $\begin{array}{l}-.081^{*} \\
(.014)\end{array}$ & - & $\begin{array}{l}.0000051^{*} \\
(.000002)\end{array}$ & - & 239 & 56.683 \\
\hline Visiting museums & $\begin{array}{c}7.079^{*} \\
(.780)\end{array}$ & $\begin{array}{c}.079^{*} \\
(.010)\end{array}$ & $\begin{array}{l}.129^{*} \\
(.036)\end{array}$ & $\begin{array}{c}.0000012^{*} \\
(1.44 \mathrm{E}-07)\end{array}$ & $\begin{array}{l}-.067^{*} \\
(.015)\end{array}$ & 一 & $\begin{array}{l}.0000046^{* *} \\
(.000002)\end{array}$ & - & 239 & 57.763 \\
\hline Off-road driving & $\begin{array}{l}8.070^{*} \\
(1.155)\end{array}$ & $\begin{array}{l}.037^{*} \\
(.015)\end{array}$ & $\begin{array}{l}.099^{* * *} \\
(.052)\end{array}$ & $\begin{array}{c}.0000012^{*} \\
(2.10 \mathrm{E}-07)\end{array}$ & $\begin{array}{l}-.027 \\
(.026)\end{array}$ & - & $\begin{array}{l}.0000041 \\
(.000008)\end{array}$ & - & 239 & 14.905 \\
\hline Biking & $\begin{array}{l}7.238^{*} \\
(.874)\end{array}$ & $\begin{array}{l}.098^{*} \\
(.011)\end{array}$ & $\begin{array}{l}.132^{*} \\
(.039)\end{array}$ & $\begin{array}{c}.0000013^{*} \\
(1.58 \mathrm{E}-07)\end{array}$ & $\begin{array}{l}-.042^{*} \\
(.016)\end{array}$ & - & $\begin{array}{l}.0000027^{*} \\
(.000002)\end{array}$ & 一 & 239 & 53.677 \\
\hline Running/jogging & $\begin{array}{l}6.913^{*} \\
(1.362)\end{array}$ & $\begin{array}{l}.103^{*} \\
(.018)\end{array}$ & $\begin{array}{l}.122^{* *} \\
(.061)\end{array}$ & $\begin{array}{c}.0000013^{*} \\
(2.46 \mathrm{E}-07)\end{array}$ & $\begin{array}{l}-.070 \\
(.026)\end{array}$ & - & $\begin{array}{l}.0000050^{*} \\
(.000002)\end{array}$ & 一 & 239 & 25.164 \\
\hline Walking & $\begin{array}{l}8.647^{*} \\
(.777)\end{array}$ & $\begin{array}{l}.075^{*} \\
(.010)\end{array}$ & $\begin{array}{l}.134^{*} \\
(.035)\end{array}$ & $\begin{array}{c}.0000013^{*} \\
(1.40 \mathrm{E}-07)\end{array}$ & $\begin{array}{l}-.062^{*} \\
(.014)\end{array}$ & - & $\begin{array}{l}.0000039^{*} \\
(.000001)\end{array}$ & - & 239 & 58.998 \\
\hline Cutting firewood & $\begin{array}{l}9.186^{*} \\
(.682)\end{array}$ & $\begin{array}{l}.018^{* *} \\
(.009)\end{array}$ & $\begin{array}{l}.112^{*} \\
(.030)\end{array}$ & $\begin{array}{l}.00000074^{*} \\
(1.21 \mathrm{E}-07)\end{array}$ & $\begin{array}{l}-.043^{*} \\
(.015)\end{array}$ & - & $\begin{array}{l}.000012^{*} \\
(.000004)\end{array}$ & - & 239 & 53.924 \\
\hline Collecting berries & $\begin{array}{l}8.255^{*} \\
(.796)\end{array}$ & $\begin{array}{l}.019 * * \\
(.011)\end{array}$ & $\begin{array}{l}.134 * \\
(.034)\end{array}$ & $\begin{array}{l}.00000092^{*} \\
(1.42 \mathrm{E}-07)\end{array}$ & $\begin{array}{l}.032 * \\
(.013)\end{array}$ & $\begin{array}{c}-.219^{* *} \\
(.023)\end{array}$ & $\begin{array}{r}.000022^{*} \\
(.000006)\end{array}$ & - & 239 & 55.490 \\
\hline Visiting prehistoric sites & $\begin{array}{l}8.736 \\
(.691)\end{array}$ & $\begin{array}{l}.021^{* *} \\
(.010)\end{array}$ & $\begin{array}{l}.071^{* *} \\
(.030)\end{array}$ & $\begin{array}{c}.0000011^{*} \\
(1.23 \mathrm{E}-07)\end{array}$ & $\begin{array}{l}-.033 \\
(.013)\end{array}$ & $\begin{array}{l}-.230^{*} \\
(.020)\end{array}$ & $\begin{array}{l}.0000027^{*} \\
(.000002)\end{array}$ & - & 239 & 77.633 \\
\hline Photography & $\begin{array}{l}7.618^{*} \\
(.834)\end{array}$ & $\begin{array}{l}.085^{*} \\
(.011)\end{array}$ & $\begin{array}{l}.114^{*} \\
(.037)\end{array}$ & $\begin{array}{c}.0000012^{*} \\
(1.50 \mathrm{E}-07)\end{array}$ & $\begin{array}{l}-.084^{*} \\
(.017)\end{array}$ & - & $\begin{array}{l}.000055^{*} \\
(.00001)\end{array}$ & - & 239 & 59.638 \\
\hline Day hiking & $\begin{array}{l}8.889^{*} \\
(.681)\end{array}$ & $\begin{array}{l}.054 \\
(.009)\end{array}$ & $\begin{array}{r}.116^{*} \\
(.029)\end{array}$ & $\begin{array}{l}.00000019^{*} \\
(1.21 \mathrm{E}-07)\end{array}$ & $\begin{array}{l}-.065^{*} \\
(.016)\end{array}$ & $\begin{array}{l}-.194^{*} \\
(.016)\end{array}$ & $\begin{array}{r}.000024^{*} \\
(.000006)\end{array}$ & - & 239 & 97.476 \\
\hline Horseback riding & $\begin{array}{l}8.780^{*} \\
(1.02)\end{array}$ & $\begin{array}{r}.050^{*} \\
(.014)\end{array}$ & $\begin{array}{c}.033 \\
(.047)\end{array}$ & $\begin{array}{c}.0000010^{*} \\
(1.86 \mathrm{E}-07)\end{array}$ & $\begin{array}{l}-.088^{*} \\
(.022)\end{array}$ & - & $\begin{array}{l}.000041^{*} \\
(.00001)\end{array}$ & $\begin{array}{l}.00059^{*} \\
(.0003)\end{array}$ & 239 & 22.402 \\
\hline Nature study & $\begin{array}{l}5.938^{*} \\
(.925)\end{array}$ & $\begin{array}{l}.063^{*} \\
(.012)\end{array}$ & $\begin{array}{r}.158^{*} \\
(.042)\end{array}$ & $\begin{array}{c}.0000011^{*} \\
(1.69 \mathrm{E}-07)\end{array}$ & $\begin{array}{l}-.068^{*} \\
(.021)\end{array}$ & - & $\begin{array}{l}.00706^{* *} \\
(.003)\end{array}$ & $\begin{array}{l}000021^{* *} \\
(.000008)\end{array}$ & 239 & 30.993 \\
\hline Backpacking & $\begin{array}{l}6.030^{*} \\
(1.467)\end{array}$ & $\begin{array}{l}.095^{*} \\
(.020)\end{array}$ & $\begin{array}{c}.081 \\
(.067)\end{array}$ & $\begin{array}{c}.0000012^{*} \\
(2.66 \mathrm{E}-07)\end{array}$ & $\begin{array}{l}-.105^{*} \\
(.035)\end{array}$ & - & $\begin{array}{l}.000062^{*} .0 \\
(.00001)\end{array}$ & $\begin{array}{l}00000076^{* * *} \\
4.44 E-08)\end{array}$ & 239 & 21.337 \\
\hline Primitive camping & $\begin{array}{r}7.320^{*} \\
(.788)\end{array}$ & $\begin{array}{l}.056^{*} \\
(.010)\end{array}$ & $\begin{array}{l}.094^{*} \\
(.035)\end{array}$ & $\begin{array}{l}.0000011^{*} \\
(1.4 \mathrm{E}-07)\end{array}$ & $\begin{array}{l}-.076^{*} \\
(.018)\end{array}$ & - & $\begin{array}{l}.000054^{*} \\
(.00001)\end{array}$ & - & 239 & 45.018 \\
\hline Wildlife observation & $\begin{array}{l}7.910^{*} \\
(.729)\end{array}$ & $\begin{array}{l}.068^{*} \\
(.010)\end{array}$ & $\begin{array}{l}.106^{*} \\
(.033)\end{array}$ & $\begin{array}{c}.0000011^{*} \\
(1.33 \mathrm{E}-07)\end{array}$ & $\begin{array}{l}-.066^{*} \\
(.017)\end{array}$ & - & $\begin{array}{l}.000026^{*} \\
(.000007)\end{array}$ & $\begin{array}{l}.00677^{*} \\
(.0022)\end{array}$ & 239 & 49.075 \\
\hline
\end{tabular}




\begin{tabular}{|c|c|c|c|c|c|c|c|c|c|c|c|}
\hline Activity & INTERCEP & INC345 P & CT18TMD & CCPOP86 & SUBEROS & PCTFARM & ROI & $\mathrm{RO} 2$ & $\mathbf{N}$ & $\begin{array}{c}\text { Ad } \\
\text { F-value }\end{array}$ & $\begin{array}{l}\text { usted } \\
R^{2}\end{array}$ \\
\hline \multicolumn{12}{|c|}{ Water } \\
\hline Pool swimming & $\begin{array}{l}3.091^{*} \\
(1.199)\end{array}$ & $\begin{array}{l}0.090^{*} \\
(.012)\end{array}$ & $\begin{array}{l}0.058^{* *} \\
(.036)\end{array}$ & $\begin{array}{l}0.0000010^{*} \\
(1.43 \mathrm{E}-07)\end{array}$ & $\begin{array}{r}-0.023^{*} \\
(.014)\end{array}$ & - & $\begin{array}{l}0.00143^{*} \\
(.0005)\end{array}$ & $\begin{array}{l}0.109^{*} \\
(.015)\end{array}$ & 239 & 58.521 & .59 \\
\hline Motorized boating & $\begin{array}{l}9.780^{*} \\
(.833)\end{array}$ & $\begin{array}{l}.032^{*} \\
(.011)\end{array}$ & $\begin{array}{l}.076^{* *} \\
(.037)\end{array}$ & $\begin{array}{l}.0000010^{*} \\
(1.44 \mathrm{E}-07)\end{array}$ & $\begin{array}{l}-.075^{*} \\
(.013)\end{array}$ & - & $\begin{array}{l}.00120^{*} \\
(.0003)\end{array}$ & $\begin{array}{c}.000219^{* *} \\
(.00009)\end{array}$ & 239 & 31.265 & .43 \\
\hline Water-sking & $\begin{array}{l}8.229 \\
(.903)\end{array}$ & $\begin{array}{l}.045^{*} \\
(.012)\end{array}$ & $\begin{array}{l}.080^{* *} \\
(.040)\end{array}$ & $\begin{array}{c}.0000011^{*} \\
(1.62 \mathrm{E}-07)\end{array}$ & $\begin{array}{l}-.054^{*} \\
(.015)\end{array}$ & - & $\begin{array}{l}.00144^{*} \\
(.0004)\end{array}$ & - & 239 & 29.446 & .37 \\
\hline Rafting/tubing & $\begin{array}{r}-12.760^{*} \\
(5.004)\end{array}$ & $\begin{array}{l}.183^{*} \\
(.066)\end{array}$ & $\begin{array}{l}.429^{* * *} \\
(.223)\end{array}$ & $\begin{array}{c}.0000012^{*} \\
(9.20 \mathrm{E}-07)\end{array}$ & $\begin{array}{l}-.041 \\
(.094)\end{array}$ & - & $\begin{array}{l}.000384^{*} \\
(.0001)\end{array}$ & $\begin{array}{l}.9553^{* *} \\
(.431)\end{array}$ & 239 & 6.431 & .12 \\
\hline Canoeing/kayaking & $\begin{array}{l}5.007^{*} \\
(.958)\end{array}$ & $\begin{array}{c}.072^{*} \\
(.013)\end{array}$ & $\begin{array}{l}.140^{*} \\
(.043)\end{array}$ & $\begin{array}{c}.0000010^{*} \\
(1.73 \mathrm{E}-07)\end{array}$ & $\begin{array}{l}-.038^{* *} \\
(.015)\end{array}$ & - & $\begin{array}{l}.01052^{*} \\
(.004)\end{array}$ & $\begin{array}{l}.00158^{*} \\
(.0006)\end{array}$ & 239 & 33.973 & .45 \\
\hline Rowing/paddling, etc. & $\begin{array}{r}6.392^{*} \\
(.919)\end{array}$ & $\begin{array}{r}.066^{*} \\
(.012)\end{array}$ & $\begin{array}{l}.123^{*} \\
(.041)\end{array}$ & $\begin{array}{c}.0000010^{*} \\
(1.66 \mathrm{E}-07)\end{array}$ & $\begin{array}{l}-.062^{*} \\
(.015)\end{array}$ & - & $\begin{array}{l}.000739^{* * *} \\
(.0003)\end{array}$ & - & 239 & 32.137 & .40 \\
\hline Stream/lake swimming & $\begin{array}{r}9 \\
9.258^{*} \\
(.827)\end{array}$ & $\begin{array}{l}.039^{*} \\
(.011)\end{array}$ & $\begin{array}{l}.104^{*} \\
(.030)\end{array}$ & $\begin{array}{c}.0000011^{*} \\
(1.41 \mathrm{E}-07)\end{array}$ & $\begin{array}{l}-.066^{*} \\
(.013)\end{array}$ & - & $\begin{array}{l}.000876^{*} \\
(.003)\end{array}$ & $\begin{array}{l}.000622^{\circ} \\
(.0002)\end{array}$ & 239 & 43.275 & .52 \\
\hline Sailing & $\begin{array}{l}3.618^{* * *} \\
(1.897)\end{array}$ & $\begin{array}{l}.117^{*} \\
(.025)\end{array}$ & $\begin{array}{l}.112 \\
(.084)\end{array}$ & $\begin{array}{c}.0000016^{*} \\
(3.30 \mathrm{E}-07)\end{array}$ & $\begin{array}{l}-.030 \\
(.030)\end{array}$ & - & $.000593^{*}$ & - & 239 & 24.238 & .33 \\
\hline \multicolumn{12}{|c|}{ Snow and Ice } \\
\hline Downhill skiing & $\begin{array}{l}11.455^{*} \\
(2.146)\end{array}$ & $\begin{array}{l}0.104^{*} \\
(.023)\end{array}$ & $\begin{array}{l}-0.162^{* * a} a \\
(.068)\end{array}$ & $\begin{array}{l}0.0000011^{*} \\
(2.74 \mathrm{E}-07)^{*}\end{array}$ & $\begin{array}{l}0.0013^{* *} \\
(.0005)\end{array}$ & $\begin{array}{c}-0.256^{\circ} \\
(.046)\end{array}$ & $\begin{array}{l}.001086^{\circ} \\
(.0003)\end{array}$ & - & 239 & 41.848 & .52 \\
\hline Cross-country skiing & $\begin{array}{l}7.570^{* *} \\
(4.061)\end{array}$ & $\begin{array}{l}.187^{*} \\
(.043)\end{array}$ & $\begin{array}{l}-.248^{* * a} \\
(.124)\end{array}$ & $\begin{array}{c}.0000014^{*} \\
(5.06 \mathrm{E}-07)\end{array}$ & $\begin{array}{l}.0025^{*} \\
(.001)\end{array}$ & - & $\begin{array}{c}.000033^{*} \\
(.000007)\end{array}$ & - & 239 & 23.268 & .32 \\
\hline Snowmobiling & $\begin{array}{c}4.963 \\
(4.730)\end{array}$ & $\begin{array}{l}.093^{* * *} \\
(.050)\end{array}$ & $\begin{array}{l}-.129 a \\
(.145)\end{array}$ & $\begin{array}{l}.00000049 \\
(5.90 \mathrm{E}-07)\end{array}$ & $\begin{array}{l}.0020^{\cdots *} \\
(.001)\end{array}$ & - & $\begin{array}{l}.000040^{*} \\
(.000008)\end{array}$ & - & 239 & 10.688 & .17 \\
\hline
\end{tabular}

"Significant at 0.01 level; " Significant at 0.05 level; "*"Significant at 0.10 level

a For these activities, age variable was MEDAGE = median age of representative county population.

Source: Cordell and Bergstrom (1989), table 3, page 20

representative county characteristics were obtained from the Bureau of the Census' City and County Databook. The functional form of the community-level recreation demand model (Cordell and Bergstrom 1991), was:

ATRIPS $^{=}=\mathrm{f}(\mathrm{P}, \mathrm{S}, \mathrm{SO}, \mathrm{Z}, \mathrm{H})$

where

$$
\begin{aligned}
& \text { ATRIPS }^{-} \text {annual trips demanded for activity } \\
& \text { k by a community } \\
& \mathrm{P}=\text { cost or price of trips for activity } \mathrm{k} \\
& \mathrm{S}=\text { suitability of sites used for activity } \mathrm{k} \\
& \mathrm{SO}=\text { substitute recreation opportunities } \\
& \text { available to a community }
\end{aligned}
$$

$$
\begin{array}{ll}
\mathrm{Z} & =\text { population } 12 \text { years old and older } \\
\mathrm{H} & =\text { characteristics }
\end{array}
$$

The estimated model for demand was:

$$
\begin{aligned}
\ln \left(\text { TRIPS }_{\mathrm{kij}}\right) & =\beta_{0}-\beta_{1} \text { PRICE }_{\mathrm{kij}}+\beta_{2} \text { INC345 }_{\mathrm{i}} \\
& +\beta_{3} \text { PCT18TMD }_{\mathrm{i}}+\beta_{4} \text { CCPOP86 }_{\mathrm{i}} \\
& -\beta_{5} \text { PCTFARM }_{\mathrm{i}}-\beta_{6} \text { SUBEROS }_{\mathrm{ki}} \\
& +\beta_{7} \text { SUIT }_{\mathrm{kj}}
\end{aligned}
$$

where TRIPS $_{\mathrm{kij}}$ is the natural log of annual trips for activity k demanded from representative county i to site $\mathrm{j}, \mathrm{PRICE}_{\mathrm{kjj}}$ is the cost of trips for activity $\mathrm{k}$ from represen- 
tative county i to site $\mathrm{j}, \mathrm{SUIT}_{\mathrm{kj}}$ is the suitability of site $\mathrm{j}$ for activity $k$, and all other variables are as defined for the consumption function, eq. [2]. ${ }^{3}$ Table 4 lists the national estimated community demand coefficients. The trip price coefficients and the regional trip price means were critical information in the calculation of maximum preferred demand (MPD) for each activity.

Differences between 1989 RPA Assessment and 1993 RPA Update.-MPD is the number of trips that households would prefer to consume, given constant trip costs and an unconstrained supply of recreation opportunities (Cordell and others 1990). Thus, MPD is a measure of recreation trip consumption similar to expected supply, but with different assumptions. The major difference is that MPD holds price constant at the base year (1987) level, while expected supply allows price to rise to the equilibrium point where the quantity of trips demanded is equal to the quantity of trips supplied. The MPD quantity of trips, therefore, results from shifts in demand and a fixed trip price. Further, no constraints are placed on recreation resources and facilities; a decrease in these would drive up trip costs. MPD uses current consumption of trips (1987) as the base-year starting off point, as does expected supply. The 1993 RPA Update improved on the MPD projections in the 1989 RPA Assessment by using regional calculations of current trip consumption, and regional trip price beta coefficients and means rather than national. A projection of MPD was calculated for each of the recreation activities in the four RPA regions, indexing the projected change back to the base year level of 100 .

\section{Projections}

\section{Expected Supply}

Current regional trip consumption estimates provide a base for regional consumption projections, which are indexed to current levels. The indices are multiplied by an estimate of the actual number of trips taken for a given activity by the entire assessment region. Trip estimates were calculated separately and are described later in this report.

\footnotetext{
${ }^{3} T o$ estimate trip costs, an allocation index was devised to allocate the reported annual activity $k$ trips, having no site information, to the PARVS sites that were used by residents of each representative county and that were suitable for that activity. Each activity, therefore, had a unique sample size because of different origindestination combinations. For a more complete discussion of the trips allocation index; see Cordell and Bergstrom (1989).
}

Regional projections of recreation trip consumption (i.e., expected supply) followed the same methods used for the 1989 RPA Assessment national-level consumption projections. Expected future levels of all independent variables were determined for five planning years: $2000,2010,2020,2030,2040$. The expected future values were derived from anticipated percentage changes which were applied to the base year mean values of the independent variables. The only exception is the substitute recreation opportunities variable, SUBEROS. This variable was held constant at the 1987 base year level throughout the planning horizon under the assumption that substitute recreation opportunities would neither increase nor decrease. Coefficients in the models were assumed to not change over time.

The same projected changes in recreation resource and facility variables that were used in the 1989 RPA Assessment were applied uniformly across the four regions. This is a recognized limitation of the 1993 RPA Update. Ideally, resource projections would reflect regional variation. Nonetheless, these projections represent best estimates of resource change based on extending recent past broad-based national trends into the future. Table 5 lists projected percentage changes in the 12 categories of recreation and wilderness resources and uses (Cordell and others 1990). Resource and facility variables from the 31 recreation consumption models were assigned the expected percentage change of the resource category in which they best fit. For example, the resource variable "backpacking land" occurs primarily in the resource category, "Wilderness and other Extensive Roadless Areas". This individual variable is expected to change at the same percentage rate as the resource category-9\% decline by $2000,15 \%$ decline by 2010 , etc.

The combination of projected future values for the independent variables and estimated coefficients allows projections of recreation consumption to the year 2040 for each activity. Regional variation in the baseline values for the independent variables and in theexpected future values of population and household income enable regional projections. A growth index is calculated for each of the five planning years with 1987 as the baselevel. Projections areexpressed in percentagechange from this base.

\section{Maximum Preferred Demand}

Regional MPD projections were developed in the same manner as the regional expected supply projections. The demand model functional form, eq. [3], was 


\begin{tabular}{|c|c|c|c|c|c|c|c|}
\hline \multirow[b]{2}{*}{ Developed camping } & \multirow[b]{2}{*}{$\begin{array}{l}4.503^{*} \\
(.330)\end{array}$} & \multicolumn{6}{|c|}{ Land } \\
\hline & & $\begin{array}{l}-0.018^{*} \\
(.0004)\end{array}$ & $\begin{array}{l}0.075^{*} \\
(.004)\end{array}$ & $\begin{array}{l}0.088^{*} \\
(.014)\end{array}$ & $\begin{array}{c}0.0000011^{*} \\
(4.43 E-08)\end{array}$ & $\begin{array}{r}-0.026 * \\
(.005)\end{array}$ & $\begin{array}{l}0.122 * \\
(.016)\end{array}$ \\
\hline Picnicking & $\begin{array}{l}4.882^{*} \\
(.400)\end{array}$ & $\begin{array}{l}-.050^{*} \\
(.0011)\end{array}$ & $\begin{array}{l}.073^{*} \\
(.005)\end{array}$ & $\begin{array}{l}.136 " \\
(.016)\end{array}$ & $\begin{array}{c}.0000014^{*} \quad- \\
(6.72 \mathrm{E}-08)\end{array}$ & $\begin{array}{l}-.027^{*} \\
(.006)\end{array}$ & $\begin{array}{l}.093^{\circ} \\
(.016)\end{array}$ \\
\hline Sightseeing & $\begin{array}{l}7.016 " \\
(.248)\end{array}$ & $\begin{array}{l}-.018 \\
(.0003)\end{array}$ & $\begin{array}{r}.029^{*} \\
(.003)\end{array}$ & $\begin{array}{l}.081^{*} \\
(.010)\end{array}$ & $\begin{array}{c}.00000088^{*}-0.180 \\
(3.30 \mathrm{E}-08)(.007)\end{array}$ & $\begin{array}{l}-.028 * \\
(.004)\end{array}$ & $\begin{array}{l}.204^{*} \\
(.010)\end{array}$ \\
\hline Family gathering & $\begin{array}{l}3.902^{*} \\
(.273)\end{array}$ & $\begin{array}{l}-.023^{*} \\
(.0004)\end{array}$ & $\begin{array}{l}.078^{*} \\
(.003)\end{array}$ & $\begin{array}{l}.131^{*} \\
(.011)\end{array}$ & $\begin{array}{c}.0000011^{\circ} \\
(3.78 \mathrm{E}-08)\end{array}$ & $\begin{array}{l}-.040 * \\
(.005)\end{array}$ & $\begin{array}{l}.146^{\circ} \\
(.012)\end{array}$ \\
\hline Pleasure driving & $\begin{array}{l}5.872^{*} \\
(.005)\end{array}$ & $\begin{array}{l}-.036 * \\
(.001)\end{array}$ & $\begin{array}{l}.076^{*} \\
(.005)\end{array}$ & $\begin{array}{l}.077^{*} \\
(.017)\end{array}$ & $\begin{array}{c}.0000013^{\circ}- \\
(6.61 \mathrm{E}-08)\end{array}$ & $\begin{array}{l}.017^{*} \\
(.007)\end{array}$ & $\begin{array}{l}.159^{\circ} \\
(.016)\end{array}$ \\
\hline Visiting historic sites & $\begin{array}{l}6.780^{*} \\
(.303)\end{array}$ & $\begin{array}{l}-.023^{*} \\
(.0005)\end{array}$ & $\begin{array}{l}.024^{*} \\
(.004)\end{array}$ & $\begin{array}{l}.088^{*} \\
(.013)\end{array}$ & $\begin{array}{r}.00000079-.200^{*} \\
(4.12 \mathrm{E}-08)(.008)\end{array}$ & $\begin{array}{l}-.039^{*} \\
(.005)\end{array}$ & $\begin{array}{l}.215^{*} \\
(.010)\end{array}$ \\
\hline $\begin{array}{l}\text { Attending special } \\
\text { events }\end{array}$ & $\begin{array}{l}4.214^{*} \\
(.317)\end{array}$ & $\begin{array}{l}-.029^{*} \\
(.0007)\end{array}$ & $\begin{array}{l}.072^{*} \\
(.004)\end{array}$ & $\begin{array}{l}.112^{*} \\
(.014)\end{array}$ & $\begin{array}{cc}.0000011^{*} & - \\
(4.77 \mathrm{E}-08) & -\end{array}$ & $\begin{array}{l}-.052^{*} \\
(.005)\end{array}$ & $\begin{array}{l}.142^{*} \\
(.015)\end{array}$ \\
\hline Visiting museums & $\begin{array}{l}5.535^{*} \\
(.367)\end{array}$ & $\begin{array}{l}-.023 \\
(.0007)\end{array}$ & $\begin{array}{l}.061^{*} \\
(.004)\end{array}$ & $\begin{array}{l}.066^{*} \\
(.016)\end{array}$ & $\begin{array}{cc}.0000011^{*} & - \\
(5.39 \mathrm{E}-08) & -\end{array}$ & $\begin{array}{l}.061^{*} \\
(.006)\end{array}$ & $\begin{array}{l}.183^{\circ} \\
(.012)\end{array}$ \\
\hline Off-road driving & $\begin{array}{l}6.877^{*} \\
(.864)\end{array}$ & $\begin{array}{l}-.044 \\
(.004)\end{array}$ & $\begin{array}{c}.013 \\
(.011)\end{array}$ & $\begin{array}{l}.083^{* *} \\
(.036)\end{array}$ & $\begin{array}{cc}.0000016^{*} & - \\
(1.82 \mathrm{E}-07) & -\end{array}$ & $\begin{array}{l}-.053^{*} \\
(.012)\end{array}$ & $\begin{array}{l}.286^{\circ} \\
(.031)\end{array}$ \\
\hline Biking & $\begin{array}{l}3.488 * \\
(.386)\end{array}$ & $\begin{array}{l}-.031^{*} \\
(.001)\end{array}$ & $\begin{array}{l}.116^{*} \\
(.005)\end{array}$ & $\begin{array}{l}.123^{*} \\
(.017)\end{array}$ & $\begin{array}{c}.0000013^{*} \quad- \\
(6.20 \mathrm{E}-08)\end{array}$ & $\begin{array}{l}-.015^{*} \\
(.006)\end{array}$ & $\begin{array}{l}.120^{*} \\
(.015)\end{array}$ \\
\hline Running/jogging & $\begin{array}{c}4.681^{*} \\
(1.100)\end{array}$ & $\begin{array}{l}-.135^{*} \\
(.014)\end{array}$ & $\begin{array}{l}.137^{*} \\
(.013)\end{array}$ & $\begin{array}{l}.070 * * * \\
(.047)\end{array}$ & $\begin{array}{c}.0000021^{*} \\
(2.46 \mathrm{E}-07)\end{array}$ & $\begin{array}{l}-.009 \\
(.017)\end{array}$ & $\begin{array}{l}.171^{\circ} \\
(.047)\end{array}$ \\
\hline Walking & $\begin{array}{l}5.001^{*} \\
(.310)\end{array}$ & $\begin{array}{l}-.027^{*} \\
(.001)\end{array}$ & $\begin{array}{l}.083^{*} \\
(.004)\end{array}$ & $\begin{array}{l}.131^{*} \\
(.013)\end{array}$ & $\begin{array}{c}.0000012^{*} \\
(4.70 \mathrm{E}-08)\end{array}$ & $\begin{array}{l}-.034^{\bullet} \\
(.005)\end{array}$ & $\begin{array}{l}.147^{\circ} \\
(.012)\end{array}$ \\
\hline Cutting firewood & $\begin{array}{l}6.820^{*} \\
(.784)\end{array}$ & $\begin{array}{l}-.032^{*} \\
(.004)\end{array}$ & $\begin{array}{l}.036^{*} \\
(.014)\end{array}$ & $\begin{array}{l}.177^{\circ} \\
(.033)\end{array}$ & $\begin{array}{c}.00000075^{*} .181^{*} \\
(1.73 \mathrm{E}-07)(.030)\end{array}$ & $\begin{array}{l}-.060^{\circ} \\
(.011)\end{array}$ & $\begin{array}{l}-.024 \\
(.037)\end{array}$ \\
\hline Collecting berries & $\begin{array}{l}5.556^{*} \\
(.800)\end{array}$ & $\begin{array}{l}-.024^{*} \\
(.003)\end{array}$ & $\begin{array}{l}.033^{*} \\
(.012)\end{array}$ & $\begin{array}{r}.232^{*} \\
(.030)\end{array}$ & $\begin{array}{c}.00000074^{*}-.196 * \\
(1.31 \mathrm{E}-07)(.028)\end{array}$ & $\begin{array}{l}-.072 \\
(.010)\end{array}$ & $\begin{array}{l}.048 \\
(.034)\end{array}$ \\
\hline Visiting prehistoric sites & $\begin{array}{l}7.595^{*} \\
(.441)\end{array}$ & $\begin{array}{l}-.026 * \\
(.001)\end{array}$ & $\begin{array}{l}.008 \\
(.006)\end{array}$ & $\begin{array}{l}-.012 \\
(.019)\end{array}$ & $\begin{array}{r}.0000012^{*}-.215^{*} \\
(7.55 \mathrm{E}-08)(.012)\end{array}$ & $\begin{array}{l}-.016 * * \\
(.007)\end{array}$ & $\begin{array}{l}.175^{\circ} \\
(.014)\end{array}$ \\
\hline Photography & $\begin{array}{l}2.967^{*} \\
(.357)\end{array}$ & $\begin{array}{l}-.022^{*} \\
(.0009)\end{array}$ & $\begin{array}{l}.094 \\
(.004)\end{array}$ & $\begin{array}{l}.122^{*} \\
(.015)\end{array}$ & $\begin{array}{c}.0000012^{*} \quad- \\
(5.37 \mathrm{E}-08)\end{array}$ & $\begin{array}{l}-.024^{*} \\
(.006)\end{array}$ & $\begin{array}{l}.198^{*} \\
(.016)\end{array}$ \\
\hline Day hiking & $\begin{array}{l}5.711^{*} \\
(.395)\end{array}$ & $\begin{array}{l}-.039^{*} \\
(.001)\end{array}$ & $\begin{array}{l}.064^{*} \\
(.005)\end{array}$ & $\begin{array}{l}.108^{*} \\
(.016)\end{array}$ & $\begin{array}{c}.0000010^{*} \\
(6.86 \mathrm{E}-08)\end{array}$ & $\begin{array}{l}.004 \\
(.006)\end{array}$ & $\begin{array}{l}.083^{*} \\
(.015)\end{array}$ \\
\hline Horseback riding & $\begin{array}{l}4.498^{*} \\
(.431)\end{array}$ & $\begin{array}{l}-.046 * \\
(.001)\end{array}$ & $\begin{array}{l}.079^{*} \\
(.005)\end{array}$ & $\begin{array}{l}.025 \\
(.019)\end{array}$ & $\begin{array}{l}.0000013^{*}- \\
(7.72 \mathrm{E}-08)\end{array}$ & $\begin{array}{l}-.037^{*} \\
(.007)\end{array}$ & $\begin{array}{l}.223^{*} \\
(.017)\end{array}$ \\
\hline Nature study & $\begin{array}{l}1.634^{*} \\
(.397)\end{array}$ & $\begin{array}{l}-.029^{*} \\
(.0009)\end{array}$ & $\begin{array}{l}.079^{*} \\
(.005)\end{array}$ & $\begin{array}{l}.156^{*} \\
(.017)\end{array}$ & $\begin{array}{c}.0000011^{*} \\
(5.84 \mathrm{E}-08)\end{array}$ & $\begin{array}{l}-.021^{\bullet} \\
(.007)\end{array}$ & $\begin{array}{l}.176 * \\
(.018)\end{array}$ \\
\hline 3ackpacking & $\begin{array}{l}3.237^{*} \\
(.515)\end{array}$ & $\begin{array}{l}-.012^{*} \\
(.001)\end{array}$ & $\begin{array}{l}.106 \\
(.006)\end{array}$ & $\begin{array}{l}-.006 \\
(.022)\end{array}$ & $\begin{array}{l}.0000013^{*}- \\
(7.59 \mathrm{E}-08)\end{array}$ & $\begin{array}{l}-.030^{*} \\
(.008)\end{array}$ & $\begin{array}{l}.279^{*} \\
(.018)\end{array}$ \\
\hline Primitive Camping & $\begin{array}{l}3.819^{*} \\
(.344)\end{array}$ & $\begin{array}{l}-.029^{*} \\
(.0007)\end{array}$ & $\begin{array}{l}.069^{*} \\
(.004)\end{array}$ & $\begin{array}{l}.072^{*} \\
(.015)\end{array}$ & $\begin{array}{c}.0000012^{*} \quad- \\
(5.20 \mathrm{E}-08)\end{array}$ & $\begin{array}{l}-.043^{*} \\
(.006)\end{array}$ & $\begin{array}{l}.236^{*} \\
(.013)\end{array}$ \\
\hline Nildlife Observation & $\begin{array}{l}5.622 \\
(.269)\end{array}$ & $\begin{array}{l}-.022 \\
(.0004)\end{array}$ & $\begin{array}{c}.051^{*} \\
(.003)\end{array}$ & $\begin{array}{l}.084^{*} \\
(.011)\end{array}$ & $\begin{array}{r}.00000089^{*}-.166 \\
(3.73 \mathrm{E}-08)(.007)\end{array}$ & $\begin{array}{l}-.008^{* *} \\
(.004)\end{array}$ & $\begin{array}{l}.181^{*} \\
(.012)\end{array}$ \\
\hline
\end{tabular}

\begin{tabular}{|c|c|c|}
\hline 3161 & 509.337 & .49 \\
\hline 2883 & 522.744 & .52 \\
\hline 4538 & 954.731 & .60 \\
\hline 4179 & 838.602 & .55 \\
\hline 2877 & 347.847 & .42 \\
\hline 3050 & 623.000 & .59 \\
\hline 3307 & 501.783 & .48 \\
\hline 2749 & 376.924 & .45 \\
\hline 800 & 55.331 & .29 \\
\hline 2998 & 434.239 & .46 \\
\hline 843 & 67.158 & .32 \\
\hline 3534 & 581.972 & .50 \\
\hline 506 & 42.010 & .36 \\
\hline 579 & 58.246 & .41 \\
\hline 1935 & 220.036 & .44 \\
\hline 3128 & 402.231 & .44 \\
\hline 2656 & 414.921 & .52 \\
\hline 2688 & 316.176 & .41 \\
\hline 3272 & 341.182 & .38 \\
\hline 2277 & 191.289 & .33 \\
\hline 2946 & 501.580 & .50 \\
\hline 3940 & 712.564 & .56 \\
\hline
\end{tabular}




\begin{tabular}{|c|c|c|c|c|c|c|c|c|c|c|}
\hline Activity & INTERCEP & PRICE $E_{i j k}$ & INC345 & PCT18TMD & \multicolumn{2}{|c|}{ CCPOP86 $_{i}$ PCTFARM $_{i}$ SUBEROS $_{k i}$} & \multirow[t]{2}{*}{ SUIT $_{k j}$} & \multirow[t]{2}{*}{$\mathbf{N}$} & \multicolumn{2}{|c|}{$\begin{array}{l}\text { Adjusted } \\
\text { F-value } R^{2}\end{array}$} \\
\hline \multicolumn{9}{|c|}{ Water } & & \\
\hline Pool swimming & $\begin{array}{l}3.136^{*} \\
(.891)\end{array}$ & $\begin{array}{r}-0.036^{*} \\
(.002)\end{array}$ & $\begin{array}{l}0.107^{*} \\
(.011)\end{array}$ & $\begin{array}{l}0.241^{*} \\
(.041)\end{array}$ & $\begin{array}{l}0.0000012^{*}- \\
(1.41 \mathrm{E}-07)\end{array}$ & $\begin{array}{l}0.024 \\
(.022)\end{array}$ & - & 857 & 131.410 & .43 \\
\hline Motorized boating & $\begin{array}{l}6.280^{*} \\
(.596)\end{array}$ & $\begin{array}{l}-.038^{*} \\
(.002)\end{array}$ & $\begin{array}{l}.061^{*} \\
(.007)\end{array}$ & $\begin{array}{l}.068^{*} \\
(.024)\end{array}$ & $\begin{array}{c}.0000014^{*} \quad- \\
(1.00 \mathrm{E}-07)\end{array}$ & $\begin{array}{l}-.033^{*} \\
(.008)\end{array}$ & $\begin{array}{l}0.155^{*} \\
(.019)\end{array}$ & 1537 & 176.174 & .41 \\
\hline Water-skiing & $\begin{array}{l}4.575^{*} \\
(.590)\end{array}$ & $\begin{array}{l}-.028^{*} \\
(.002)\end{array}$ & $\begin{array}{l}.067^{*} \\
(.007)\end{array}$ & $\begin{array}{l}.062^{*} \\
(.024)\end{array}$ & $\begin{array}{c}.0000014^{*} \quad- \\
(9.56 \mathrm{E}-08)\end{array}$ & $\begin{array}{l}-.012 \\
(.008)\end{array}$ & $\begin{array}{l}.187^{*} \\
(.019)\end{array}$ & 1553 & 136.448 & .34 \\
\hline Rafting/tubing & $\begin{array}{l}4.653^{*} \\
(.816)\end{array}$ & $\begin{array}{l}-.033^{*} \\
(.002)\end{array}$ & $\begin{array}{l}.064^{*} \\
(.009)\end{array}$ & $\begin{array}{l}.064^{* * *} \\
(.036)\end{array}$ & $\begin{array}{l}.0000014^{*}- \\
(1.28 \mathrm{E}-07)\end{array}$ & $\begin{array}{l}.006 \\
(.012)\end{array}$ & $\begin{array}{l}.300^{*} \\
(.030)\end{array}$ & 1379 & 96.158 & .29 \\
\hline Canoeing/kayaking & $\begin{array}{l}1.285 \\
(.442)\end{array}$ & $\begin{array}{l}-.048^{*} \\
(.001)\end{array}$ & $\begin{array}{l}.087^{*} \\
(.005)\end{array}$ & $\begin{array}{l}.167^{*} \\
(.019)\end{array}$ & $\begin{array}{c}.0000013^{*} \text { - } \\
(7.61 \mathrm{E}-08)\end{array}$ & $\begin{array}{l}-.019^{*} \\
(.006)\end{array}$ & $\begin{array}{l}.250^{*} \\
(.016)\end{array}$ & 2381 & 455.052 & .53 \\
\hline Rowing/paddling, etc. & $\begin{array}{l}2.066^{*} \\
(.418)\end{array}$ & $\begin{array}{l}-.024^{*} \\
(.001)\end{array}$ & $\begin{array}{l}.074^{*} \\
(.005)\end{array}$ & $\begin{array}{l}.124^{*} \\
(.019)\end{array}$ & $\begin{array}{r}.000013^{*} \quad- \\
(7.39 \mathrm{E}-08)\end{array}$ & $\begin{array}{l}-.023^{*} \\
(.006)\end{array}$ & $\begin{array}{l}.221^{*} \\
(.015)\end{array}$ & 2413 & 248.152 & .38 \\
\hline Stream/lake swimming & $\begin{array}{l}6.100^{*} \\
(.399)\end{array}$ & $\begin{array}{l}-.034^{*} \\
(.0007)\end{array}$ & $\begin{array}{l}.057^{*} \\
(.005)\end{array}$ & $\begin{array}{l}.077^{*} \\
(.017)\end{array}$ & $\begin{array}{c}.0000011^{*}- \\
(5.68 \mathrm{E}-08)\end{array}$ & $\begin{array}{l}-.035^{*} \\
(.006)\end{array}$ & $\begin{array}{l}.183^{*} \\
(.013)\end{array}$ & 2678 & 521.61 & .54 \\
\hline Sailing & $\begin{array}{r}-0.453 \\
(.857)\end{array}$ & $\begin{array}{l}-.028^{*} \\
(.0020)\end{array}$ & $\begin{array}{l}.134^{*} \\
(.010)\end{array}$ & $\begin{array}{l}.158^{*} \\
(.035)\end{array}$ & $\begin{array}{l}.0000019^{*}- \\
(1.44 \mathrm{E}-07)\end{array}$ & $\begin{array}{l}-.016 \\
(.012)\end{array}$ & $\begin{array}{l}.126^{*} \\
(.028)\end{array}$ & 1541 & 129.71 & .33 \\
\hline \multicolumn{11}{|c|}{ Snow and lce } \\
\hline Downhill skiing & $\begin{array}{l}7.765^{*} \\
(2.43)\end{array}$ & $\begin{array}{r}-0.031^{*} \\
(.005)\end{array}$ & $\begin{array}{l}0.059^{* *} \\
(.028)\end{array}$ & $\begin{array}{l}0.135^{* * *} \\
(.083)\end{array}$ & $\begin{array}{l}0.00000037^{*}-0.368^{*} \\
(1.80 \mathrm{E}-07) \quad(.081)\end{array}$ & $\begin{array}{l}0.001^{* *} \\
(.0005)\end{array}$ & - & 138 & 22.706 & 0.49 \\
\hline Cross-country skiing & $\begin{array}{l}1.185 \\
(1.08)\end{array}$ & $\begin{array}{l}-.034^{*} \\
(.002)\end{array}$ & $\begin{array}{l}.216^{*} \\
(.009)\end{array}$ & $\begin{array}{l}-.130^{*} \\
(.033)\end{array}$ & $\begin{array}{l}.0000015^{*} \quad- \\
(1.27 \mathrm{E}-07)\end{array}$ & $\begin{array}{l}.002^{*} \\
(.002)\end{array}$ & $\begin{array}{l}.338^{*} \\
(.037)\end{array}$ & 2656 & 231.917 & .34 \\
\hline Snowmobiling & $\begin{array}{l}-3.452^{*} \\
(0.787)\end{array}$ & $\begin{array}{l}-.026^{*} \\
(.002)\end{array}$ & $\begin{array}{l}.146^{*} \\
(.010)\end{array}$ & $\begin{array}{l}.091^{*} \\
(.035)\end{array}$ & $\begin{array}{l}.00000089^{*}- \\
(1.41 \mathrm{E}-07)\end{array}$ & $\begin{array}{l}.172^{*} \\
(.009)\end{array}$ & $\begin{array}{l}.095^{* *} \\
(.042)\end{array}$ & 2664 & 157.859 & .26 \\
\hline
\end{tabular}

"Significant at 0.01 level; "*Significant at 0.05 level; "** Significant at 0.10 level.

Source: Cordell and Bergstrom (1989), table 1, page 17

specified nationally for each activity based on all of the origin-destination combinations in the PARVS data set. From the demand models, trip cost coefficients and the mean value of trip costs were used in a formula to calculate MPD from current trip consumption. MPD is the upper limit of trips that households would consume if the cost of a recreation trip was not allowed to increase and available opportunities for recreation were not a limiting factor. Future values of MPD, then, depend primarily on shifts in demand resulting from changes in other determinants, such as age, income, and population. Multiplying the projection indices by the base year number of trips produces an estimate of the absolute change in the total number of trips.

\section{Estimates of Recreation Trips}

In addition to projection indices of recreation trip consumption, the absolute number of current trips from each region for each activity was estimated. The estimate was the product of the following four values: (1) regional estimates of the 1987 population age 12 years and older, (2) the national percentage of the population at least 12 years old who participate in the activity, (3) the percentage of trips for the activity that occur away from home, and (4) mean annual activity trips per person, by region. Regional population estimates (1987) were developed from U.S. Census Bureau data. Regional estimates of population at least 12 years old are: North, 98.7 
Table 5.-Estimated future trends in land, water, and snow and ice resources and environments if recent trends (1970-1987) in amounts of resources available for outdoor recreation were to continue.

Projected percentage change from 1987

\begin{abstract}
Resources and environments
\end{abstract}

$\begin{array}{lllll}2000 & 2010 \quad 2020 \quad 2030 \quad 2040\end{array}$

\section{Land}

Wilderness and other extensive roadless areas Undeveloped areas near roads

Partially developed. roaded areas

Intensively developed sites

$\begin{array}{lllll}-9 & -15 & -21 & -26 & -31 \\ -12 & -20 & -28 & -35 & -41 \\ -9 & -15 & -21 & -26 & -31 \\ 8 & 15 & 22 & 29 & 37\end{array}$

Water

Wild and remote lakes and streams

Lakes and streams near roads

Lake and stream sites adjoined by roads

Intensively developed water sites

\section{Snow and lce}

Wilderness and other roadless areas Undeveloped areas near roads

Partially developed. roaded areas

Intensively developed winter sports sites

$\begin{array}{lllll}-9 & -15 & -21 & -26 & -31 \\ -12 & -20 & -28 & -35 & -41 \\ -9 & -15 & -21 & -26 & -31 \\ 17 & 28 & 36 & 43 & 49\end{array}$

$\begin{array}{rrrrr}3 & 6 & 8 & 9 & 10 \\ -3 & -4 & -6 & -8 & -10 \\ 8 & 15 & 22 & 29 & 37 \\ 12 & 23 & 34 & 47 & 61\end{array}$

Source: Cordell and others (1990)

million; Pacific Coast, 29.6 million; Rocky Mountains, 15.4 million; South, 64.6 million. Participation estimates were taken from the 1982-1983 Nationwide Recreation Survey (U.S. Department of the Interior 1986). Proportion of trips away from home were estimated by an expert panel of researchers. "Away from home" denotes any trip requiring motorized travel from an individual's permanent residence. Mean annual activity trips per person by region were calculated from PARVS data. Projected recreation consumption indices together with estimates of base year trips provide a projection of the expected supply of activity trips in each region (i.e., the equilibrium number of trips consumed where no shortages or surpluses of recreation supply occur).
Table 6.-Key variables identified for updating in the National Outdoor Recreation Supply Information System (NORSIS) database for the 1993 RPA Assessment Update.

\begin{tabular}{|c|c|}
\hline Variable & Description \\
\hline FEDWILD * & Acres of federally designated wilderness areas \\
\hline FEDOVER3 * & Acres of federal lands $>3$ miles from roads \\
\hline STWILDS & Acres of state designated wilderness areas \\
\hline SPOVER3 & Acres of state park lands $>3$ miles from roads \\
\hline FEDHALF3 & Acres of federal lands $1 / 2$ to 3 miles from roads \\
\hline NTFOOTMI * & Miles of National Recreation Trails (NRT) for hiking \\
\hline NTHORSMI * & Miles of NRT's for horseback use \\
\hline SPHALF3 & Acres of state park lands $1 / 2$ to 3 miles from roads \\
\hline FEDINHLF & Acres of federal lands within $1 / 2$ mile of roads \\
\hline NTBIKEMI * & Miles of NRT available for bicycle use \\
\hline NTMOTOMI * & Miles of NRT available for motorcycle use \\
\hline FEDROADI & Miles of roads on USFS, NPS and FWS lands \\
\hline SFRECAC & Acres of state forest open for recreation \\
\hline SPINHALF & Acres of state park lands within $1 / 3$ mile of roads \\
\hline FGLACRE & Acres of state fish and game lands \\
\hline PLOPAC4 & $\begin{array}{l}\text { Acres of non-industrial, private (NIP) lands open to } \\
\text { the public, free or fee, in tracts } 500-2499 \text { acres }\end{array}$ \\
\hline PLOPAC5 & $\begin{array}{l}\text { NIP land acres open to public, in tracts of } 2500+ \\
\text { acres }\end{array}$ \\
\hline FORINDAC & Acres of forest industry owned lands \\
\hline & Acres of rural, non-federal roads and railroads \\
\hline PLOPAC23 & $\begin{array}{l}\text { NIP land acres open to public, in tracts } 20-499 \\
\text { acres }\end{array}$ \\
\hline FEDROAD2 & Miles of COE and TVA roads \\
\hline NUMRESRT & Number of commercial resorts \\
\hline TRSTACCM & Number of tourist accommodation businesses \\
\hline ZOOS & Number of zoos \\
\hline GOLFCRSE & Number of public and private golf courses \\
\hline GEDCGS & Number of federal government campgrounds \\
\hline STCGS & Number of state government campgrounds \\
\hline LOCGS & Number of local government campgrounds \\
\hline FEDRIVMI * & $\begin{array}{l}\text { Miles of federal rivers, designated or under study } \\
\text { for Wild and Scenic designation }\end{array}$ \\
\hline CANOOUTF & Number of canoe outfitters \\
\hline RUNWATR & Acres of water in rivers \\
\hline CANOERNT & $\begin{array}{l}\text { Number of canoe livery and rental firms in the } \\
\text { county }\end{array}$ \\
\hline STRIVMI & $\begin{array}{l}\text { Miles of rivers designated by states as being } \\
\text { significant for historic, cultural, scenic or recre- } \\
\text { ational reasons }\end{array}$ \\
\hline FLATWATR & Acres of water bodies \\
\hline BEACHMI & Miles of publicly accessible beach \\
\hline MARINAS & Number of marinas \\
\hline PUBPOOLS & Number of swimming pools open to the public \\
\hline PVTPOOLS & Number of swimming pools open to members \\
\hline EFSHPIER & Number of fishing piers \\
\hline VTFH & Vertical transport feet per hour at ski areas \\
\hline PLOPIAC 1 & $\begin{array}{l}\text { Acres of NIP lands open to recreation (NOT } \\
\text { leased) in tracts } 20-99 \text { acres }\end{array}$ \\
\hline PLOP2ACl & $\begin{array}{l}\text { Acres of NIP lands open to recreation (Leased) in } \\
\text { tracts } 20-99 \text { acres }\end{array}$ \\
\hline PVCGS & Number of private campgrounds (Rand McNally) \\
\hline PLFORAC & $\begin{array}{l}\text { Total acres of non-industrial private lands F land in } \\
\text { the county }\end{array}$ \\
\hline DAILYCAP & Daily lift capacity \\
\hline LOTOLAC & $\begin{array}{l}\text { Total number of acres of local government } \\
\text { recreation land (MACPARS) }\end{array}$ \\
\hline
\end{tabular}

*Indicates values were actually updated.

Source: National Outdoor Recreation Supply Information System (NORSIS) database, USDA Forest Service, Athens, GA. 


\section{RECREATION OPPORTUNITY INDICES}

\section{Updating Key Variables}

In updating the Effective Recreation Opportunity Set (EROS) indices, an attempt was made to update key supply variables within the National Outdoor RecreationSupply InformationSystem (NORSIS). The NORSIS database contains county-level data for more than 400 variables relevant to outdoor recreation supply, and was created during the period 1985-1988. Key variables were determined to be those which were important to a recreationenvironment, consumption, or demand mod$\mathrm{el}$, and had a potential to have changed significantly since 1988 (table 6). However, time and budget constraints for completing this report allowed values for only seven variables to be updated. Those included designated federal wilderness acres (FEDWILD), federal lands more than 3 miles from roads (FEDOVER3), miles in the National Wild and Scenic River System (FEDRIVMI), and miles of National Recreation Trails for use in hiking (NTFOOTMI), horseback riding (NTHORSMI), bicycling (NTBIKEMI), and motorcycling (NTMOTOMI).

Changes in wilderness area data were supplied by theForestService (FS) and Bureau of Land Management (BLM). Data on FS wilderness acres were obtained from regional Land Area Resource (LAR) reports. These reports list actual or estimated acreage by county for each wilderness area within the region. BLM data were obtained from a national database in their Washington, D.C. office, and from state maps showing the location of wilderness areas and study areas. Several National Park Service(NPS) units were added to the system since 1988. Acreage information for these areas also was obtained from BLM sources. In cases where it was not possible to directly obtain county level estimates, a grid-based sampling method was used to estimate wilderness acreage in a specific county. The sampling process was repeated three times, and the mean of the observations was used.

NPS provided updated information on National Recreation Trails, through the National Recreation Trail Guide. NPS staff provided updates in both additions and deletions to the system, and corrections to errors in the 1988 Guide. In general, few changes have occurred since 1988. Most of the changes involved previous error corrections. NPS also was the primary source for updates for Wild and Scenic River System miles. To get county-level mileage estimates, a small distance mea- suring wheel was traced along maps to derive mileage estimates. The measuring process was repeated three times, and the mean of the observations was used.

\section{Regional EROS Calculation}

Regional EROS measures extend national indices developed for the 1989 RPA Assessment by accounting for regional differences in travel distance thresholds. Before explaining the steps taken for the regionalization, a brief review of the steps taken for EROS calculation is presented. More detailed discussion is provided in English and Cordell (1993), and Cordell and others (1989). EROS is an index of the amount and location of publicly available recreation resources, facilities, and services relative to the number and location of population. EROS values corresponding to the 12 types of recreation environments were produced for each of the 239 representative counties. These environments represent resources arrayed by distance from the nearest road passable to a two-wheel-drive passenger vehicle, for the major categories of land, water, and snow and ice resources.

The primary data source for EROS calculations was the National Outdoor Recreation Supply Information System (NORSIS). For each recreation environment, an expert panel of researchers identified specific recreation resources that were either integral and essential components of that environment's resource base, or relevant, but not critical, to that recreation environment. Selected resources were assigned weights of 3 and 1 , respectively.

The first step was to compute the relative abundance of the resources in each environment for each county. Resources were first transformed to resources per capita, to account for congestion effects. Resulting values were indexed to the 95th percentile of national resource per capita values. For each environment, a weighted average of relevant resource per population indices was computed, and the result was re-indexed over all counties to the national maximum. Theresult yielded Weighted Opportunity Set Indices (WOSI) for each of the 239 representative counties.

Next, WOSI values were transformed to reflect intercounty use pressures and account for travel distance effects. Data collected in the Public Area Recreation Visitors Study (PARVS) were used to obtain relevant travel distances (RTD) specific to each of the twelve recreation settings. These mileage figures reflect the distance within which $75 \%$ of the PARVS respondents 
traveled for activities occurring in that setting. The 75\% level was used to eliminate households taking long vacations. Effectiveness of resources was assumed to decline linearly with distance, and to vanish at the threshold distance. Distances between counties were measured from county centers. The effectiveness decay weight $\left(E W_{i}\right)$ for each opportunity set $i$ between any two counties $x$ and $y$ was calculated as:

$$
\begin{gathered}
E W_{i x y}=1-\left(D_{x y} / T D_{i}\right) \text {, where } D_{x y}<=T D_{i} \\
E W_{i x y}=0 \text {, where } D_{x y}>T D_{i}
\end{gathered}
$$

where

$\mathrm{D}_{\mathrm{xy}}=$ distance between counties $\mathrm{x}$ and $\mathrm{y}$

$\mathrm{TD}_{\mathrm{i}}=$ threshold distance for opportunity set $\mathrm{i}$.

EROS values were computed as:

$$
\text { EROS }_{\mathrm{ix}}=\frac{\sum_{\mathrm{y}=1}^{\mathrm{n}}\left(\text { WOSI }_{\mathrm{iy}}{ }^{*} \mathrm{EW}_{\mathrm{ixy}}\right)}{\sum_{y=1}^{\mathrm{n}} E W_{\mathrm{ixy}}}, \mathrm{i}=1,2, \ldots 12
$$

where

$$
\begin{aligned}
\text { EROS }_{\mathrm{ix}}= & \begin{array}{l}
\text { EROS value of recreation environment } \mathrm{i} \\
\text { for county } \mathrm{x}
\end{array} \\
\text { WOSI }_{\mathrm{iy}}= & \begin{array}{l}
\text { WOSI value of recreation environment } \mathrm{i} \\
\text { for county } \mathrm{y}
\end{array} \\
\mathrm{EW}_{\mathrm{ixy}}= & \begin{array}{l}
\text { effectiveness decay weights between } \\
\text { counties y and } \mathrm{x} \text { for recreation environ- }
\end{array} \\
\mathrm{ment} \mathrm{i} . & \\
\mathrm{n} & \begin{array}{l}
\text { Number of counties whose centroids are } \\
\text { within } \mathrm{RTD}_{\mathrm{i}} \text { of county } \mathrm{x} .
\end{array}
\end{aligned}
$$

EROSvalueswerecalculated regionally by using regional relevant travel distances in the final step of EROS calculation. For the first eight recreation environments, an expanded PARVS data set was used to derive regional distances. Respondents were assigned a region based on their home location. As was done in 1989, the travel distance at the 75th percentile of respondents for each activity environment determined the regional RTD. For winter recreation environments, RTD's used in the 1989 Assessment were also used for regional distances because of an insufficient number of cases in the PARVS data set.

The inclusion of regional distances changes the previous EROS model only slightly. Effectiveness weights are now computed as:

$$
\begin{gathered}
E W_{i x y}=1-\left(D_{x y} / T_{i}^{r}\right) \text { if } D_{x y}<=T D_{i}^{r} \\
E W_{i x y}=0 \text { if } D_{x y}>T D_{i}^{r}
\end{gathered}
$$

where

$$
\begin{aligned}
\mathrm{D}_{\mathrm{xy}}= & \text { distance between counties } \mathrm{x} \text { and } \mathrm{y} \\
\mathrm{TD}_{\mathrm{i}}^{\mathrm{r}}= & \text { threshold distance for opportunity set } \mathrm{i} \\
& \text { in region } \mathrm{r} \text {, where } \mathrm{r} \text { is region of county } \mathrm{x} .
\end{aligned}
$$

The formula for EROS value computation (eq. [5]) is unchanged.

\section{Projections}

The 1989 RPA Assessment included effective recreation supply projections for the years 2000-2040. These projections used 1987 as a baseline index. Information for assumed futures was obtained from several sources. Population projections were based on Wharton Econometrics projections. Exogenous land use and recreation resource availability projections were based on past trends, mostly since 1970. Exogenous economic influences and public finance projections were assumed to remain constant (Cordell and others 1989). After estimating future values for the variables, EROS calculation methods as discussed previously were applied.

Methods for projecting regional EROS values were similar to those used in 1989. The major difference was that the current set of projections were calculated using regional population projections. For resource availability, the same resource trend projections used in the 1989 Assessment were applied to each RPA Assessment region to develop projected WOSI values. The methods described earlier to calculate EROS values from WOSI values then were applied for each projected year from 2000-2040. Projections are based on resource trends from 1970 to 1987 and projected changes in U.S. population.

\section{RESULTS}

\section{REGIONAL POPULATION COMPARISONS}

\section{Current Situation}

Population statistics describing the sample of representative counties are presented in table 7. For each of the model independent variables-population, income, age, and rural/urban residence-the representative county national mean is the mean of the four Assessment regions weighted by the number of aggregate observations. The Pacific Coast population mean is heavily influenced by the presence of representative counties in metropolitan southern California and the 
Means (standard deviation)

\begin{tabular}{|c|c|c|c|c|c|c|c|c|c|}
\hline \multirow{2}{*}{$\begin{array}{l}\text { Assessment region } \\
\text { North }\end{array}$} & \multirow{2}{*}{$\begin{array}{c}\text { Number of } \\
\text { representative } \\
\text { counties }\end{array}$} & \multicolumn{2}{|c|}{$\begin{array}{c}\text { Total } \\
\text { population }\end{array}$} & \multicolumn{2}{|c|}{$\begin{array}{c}\text { Total } \\
\% \text { households with } \\
\text { income }>\$ 30,000\end{array}$} & \multicolumn{2}{|c|}{$\begin{array}{l}\text { Total } \\
\% \text { population } \\
\text { ages } 18-32\end{array}$} & \multicolumn{2}{|c|}{$\begin{array}{l}\text { Total } \\
\% \text { population } \\
\text { living on farms }\end{array}$} \\
\hline & & 330,922 & $(640,966)$ & 26.40 & $(9.35)$ & 21.76 & $(2.01)$ & 4.67 & $(5.10)$ \\
\hline Pacific Coast & 22 & 834,614 & $(1,432,827)$ & 29.27 & $(7.88)$ & 22.36 & $(2.04)$ & 1.96 & (2.13) \\
\hline Rocky Mountains & 37 & 158,371 & $(283,237)$ & 23.81 & $(7.24)$ & 20.22 & (3.35) & 4.60 & $(4.94)$ \\
\hline South & 88 & 212,594 & $(343,106)$ & 19.06 & $(7.37)$ & 21.30 & $(2.31)$ & 3.27 & $(2.87)$ \\
\hline National & 239 & 307,005 & $(652,750)$ & 23.56 & $(8.96)$ & 21.41 & $(2.43)$ & 3.89 & $(4.22)$ \\
\hline
\end{tabular}

Source: Outdoor Recreation and Wilderness Assessment Research, USDA Forest Service, Athens, GA.

San Francisco Bay area. The much larger population mean for the Pacific Coast region is inconsequential in the projections methodology, because each region is analyzed separately. For each variable, the regional means listed in table 7 are the population characteristics from which expected future values are calculated by multiplying these figures by expected future growth rates. Because aggregate observations are, in part, a function of population, the eastern regions have a greater number of aggregate observations.

\section{Projected Changes}

Expected percentage growth rates of population and income varied by region (table 8). The Bureau of Economic Analysis (BEA) provided regional population projections (U.S. Department of Commerce 1991). These were applied to the population age 12 years and older (CCPOP86). BEA also provided projections of total personal income by region. These were divided by population projections to produce projections of per capita income. Regional projections of per capita income growth were used instead of information on the percentage of households earning at least $\$ 30,000$ annually. It was assumed that the proportion of these households in the economy would grow at about the same rate as per capita income growth.

Regional projections of percentage growth were not available for the age (PCT18TMD) and residence (PCTFARM) variables, nor for any of the recreation resource and facility variables. Expected national growth rates for these variables, as used in the 1989 RPA Assessment, were applied to the regional consumption projec- tions. It should be noted that the representative county average percent of population living on farms in 1987 was just 3.9\%, therefore, changes in this relatively small base year mean percentage show a deceptively large percentage change. For example, the percentage of the population living on farms is projected to decline to $3.0 \%$ in 2000 which is a $22.9 \%$ decrease from $3.9 \%$ in 1987 .

\section{RESOURCE OPPORTUNITIES}

\section{Key Variable Updates}

Significant changes to the National Wilderness Preservation System have occurred since 1987 (table 9). More than 5 million acres have been added to the system during that time. Of this amount, 1,086,698 acres have been added in the ForestService, 1,693,148 in the National Park Service, 1,127,088 in the Bureau of Land Management, and 1,343,444 in the Fish and Wildlife Service. There are 95 million acres in the entire National Wilderness Preservation System. Of this total, 57.4 million acres, or more than $60 \%$, are in Alaska.

In total, 1619 miles of rivers have been added to the Wild and Scenic River system since 1987 (table 10). Of these additions, 983 miles are managed by the Forest Service, 17 miles by the National Park Service, 602 miles by the Bureau of Land Management, and 17 miles by the State of Illinois. After including all of these updates, the amount of resources and facilities within 120 miles of each representative county were calculated. This resource amount was used in the consumption models as a facility measure. 


\begin{tabular}{|c|c|c|c|c|c|c|}
\hline \multirow{2}{*}{$\begin{array}{l}\text { Variable } \\
\text { and } \\
\text { region }\end{array}$} & \multirow[b]{2}{*}{$\begin{array}{c}1987 \\
\text { Base }^{2}\end{array}$} & \\
\hline & & 2000 & 2010 & 2020 & 2030 & 2040 \\
\hline \multicolumn{7}{|l|}{ Population ${ }^{3}$} \\
\hline North & 330.9 & 4.1 & 8.3 & 12.2 & 13.2 & 14.2 \\
\hline Pacific Coast & 834.6 & 24.7 & 35.0 & 41.8 & 44.4 & 46.9 \\
\hline Rocky Mountains & 158.4 & 15.9 & 23.9 & 30.0 & 32.2 & 34.5 \\
\hline South & 212.6 & 10.4 & 16.3 & 21.5 & 23.2 & 24.9 \\
\hline \multicolumn{7}{|l|}{ Income 4} \\
\hline North & 26.4 & 13.3 & 22.7 & 29.9 & 31.7 & 33.1 \\
\hline Pacific Coast & 29.3 & 12.4 & 21.5 & 29.1 & 37.9 & 46.1 \\
\hline Rocky Mountains & 23.8 & 16.0 & 27.2 & 35.5 & 45.1 & 54.0 \\
\hline South & 19.1 & 16.3 & 27.7 & 36.1 & 45.7 & 54.6 \\
\hline \multicolumn{7}{|l|}{ Age $^{5}$} \\
\hline National & 21.4 & -6.5 & -9.8 & -12.6 & -14.0 & -15.0 \\
\hline \multicolumn{7}{|l|}{ Residence $^{6}$} \\
\hline National & 3.9 & -22.9 & -38.3 & -53.7 & -69.2 & -84.6 \\
\hline
\end{tabular}

\footnotetext{
IPercentage growth rates by region were not available for the age and residence variables. National rates of percentage growth were applied uniformly across the four regions.

21987 base represents the independent variable mean values for the representative counties within each region.

${ }^{3}$ CCPOP86-population age 12 \& older in 1000's.

${ }^{4}$ INC345-percent of households with more than $\$ 30,000$ annual income.

5 PCT18TMD-percent of population between ages 18 and 32.

6PCTFARM-percent of population living on farms.

Sources: 1989 RPA Assessment of Outdoor Recreation and Wilderness. USDA Forest Service. Athens, GA. U.S. Department of Commerce (1991).
}

\section{Regional EROS Indices}

Regional EROS values provide a more accurate perspective of effective recreation opportunities. By incorporating relevant travel distances on a regional basis, variations in travel behavior are reflected in the final EROS values. Based on PARVS data, the greatest variation in relevant recreation travel distances exist between the Rocky Mountain region and all other regions (table 11). Specifically, the travel distances were significantly higher in this region for EROS categories four through eight. This may result from greater distances separating recreation areas and the recreating public. In contrast, the South region tended to show shorter relevant travel distances than the other regions for most of the EROS categories.

Table 12 shows means of the EROS indices, both by region and for the nation as a whole. A significant amount of regional variation is masked by simply looking at the national EROS mean alone. In general, effec- tive recreation opportunities are much greater in the Pacific Coast and Rocky Mountain regions for many EROS categories than in the North and South regions, as expected given the presence of more land for recreation in the West and less population pressure.

Within the land-based categories, effective wilderness opportunities (EROS 1) average about 22 times greater opportunity for people living in the Pacific Coast and Rocky Mountain regions than in the North and South regions. This result reflects the regional distribution of acreage of wilderness resources, as well as the greater numbers of people competing for these resourcesin the East. "Extensive undeveloped areas near roads" (EROS 2) and "roaded and partially developed areas" (EROS 3) show similar, but not as extreme variation. "Developed sites" (EROS 4) show a more balanced distribution. For all four land recreation environments, the Rocky Mountain Region has the greatest amount of effective recreation opportunities. 
Opportunities among water-based environmentsalso vary across regions. The greatest opportunities exist in the Rocky Mountain region for recreation on "Wild and Scenic or other remote lakes and streams" (EROS5) and "lakes or streams near roads" (EROS 6), and in the Pacific Coast region for "lake/stream sites adjoined by roads" (EROS 7) and "developed water sites" (EROS 8). Although one of these two regions tended to provide the greatest effective opportunities for each of the four water-based environments, the western regions did not dominate the opportunities as they did with land-based opportunities. For example, although the Rocky Moun-

Table 9.-National Wilderness Preservation System (NWPS) acreage (in thousands of acres) in 1987 and 1992, and growth (1987-1992), by managing agency.

\begin{tabular}{lrrr}
\hline Managing agency & 1987 & 1992 & $\begin{array}{c}\text { Change } \\
\text { since 1987 }\end{array}$ \\
\hline & & & \\
USDA Forest Service & 32,549 & 33,636 & 1,087 \\
National Park Service & 37,385 & 39,078 & 1,693 \\
Bureau of Land Management & 484 & 1,611 & 1,127 \\
Fish and Wildlife Service & 19,333 & 20,676 & 1,343 \\
Total & 89,751 & 95,001 & 5,250 \\
\hline
\end{tabular}

Source: National Outdoor Recreation Supply Information System (NORSIS) database, USDA Forest Service, Athens, GA. tain region provided the most effective opportunity for "lakes and streams near roads" (EROS 6), the Pacific Coast region had the least opportunity in this environment. The reverse was true for opportunities involving "developed water sites" (EROS 8). Of the four waterbased opportunity environments, "Wild and Scenic or other remote lakes and streams" (EROS 5) is the most balanced across regions.

Similarly, analysis of the snow and ice-based environments revealed far more opportunity in the western regions than in the eastern regions. For "wilderness and other remote back-country" (EROS9), opportunities are

Table 10.-National Wild and Scenic River system mileage in 1987 and 1992, and growth (1987-1992), by managing agency.

\begin{tabular}{lrrr}
\hline Managing agency & 1987 & 1992 & $\begin{array}{c}\text { Change } \\
\text { since 1987 }\end{array}$ \\
\hline & & & \\
USDA Forest Service & 2,570 & 3,553 & 983 \\
National Park Service & 2,015 & 2,032 & 17 \\
Bureau of Land Management & 2,437 & 3,039 & 602 \\
Fish and Wildlife Service & 1,043 & 1,043 & 0 \\
State of Illinois & 0 & 17 & 17 \\
Total & 8,065 & 9,684 & 1,619 \\
& & & \\
\hline
\end{tabular}

Source: National Outdoor Recreation Supply Information System (NORSIS) database, USDA Forest Service, Athens, GA.

Table 11.-Relevant travel distances in miles, by recreation environment and region. ${ }^{1}$

\section{Resources and environments}

North

Pacific Rocky

Coast Mountain South National

Land

EROS1: Wilderness and other extensive roadless areas

EROS2: Undeveloped areas near roads

EROS3: Partially developed, roaded areas

EROS4: Intensively developed sites

\section{Water}

EROS5: Wild \& remote lakes/streams

EROS6: Lakes/streams near roads

EROS7: Lake/stream sites adjoined by roads

EROS8: Intensively developed water sites

\section{Snow and $\mathrm{Ice}^{2}$}

EROS9: Wilderness \& other roadless areas

EROS10: Undeveloped areas near roads

EROS 11: Partially developed, roaded areas

EROS12: Intensively developed winter sports sites

100
75
95
95

95
95
95
100

$\begin{array}{rll}75 & 65 & 80 \\ 75 & 75 & 75 \\ 90 & 65 & 80 \\ 140 & 70 & 95\end{array}$

$85 \quad 75 \quad 130$

70

55

45

65

85

45

140

140

140

$\begin{array}{ll}50 & 80 \\ 40 & 60 \\ 40 & 50 \\ 35 & 40\end{array}$

100
100
200
250

100
100
200
250

\section{0}

$$
100
$$$$
200
$$

250
80
75
80
95


80
60
50
40 (1)

95
80 0

\footnotetext{
'Relevant travel distances are those distances (rounded to nearest 5 miles) within which 75\% of PARVS respondents traveled for activities occurring in that setting effectively eliminating vacation trips.

2 Relevant travel distances did not vary by region for the four snow and ice EROS categories because of an insufficient number of survey observations.
}

Source: Public Area Recreation Visitor Study (PARVS), 1985-1989. 
Land

EROS1:

EROS2:

EROS3:

EROS4:

Wilderness and other extensive roadless areas

Undeveloped areas near roads

Partially developed, roaded areas

Intensively developed sites

Water

Wild \& remote lakes/streams
EROS5:

EROS6:

EROS7:

EROS8:

Lakes/streams near roads

Lake/stream sites adjoined by roads

Intensively developed water sites

Snow and lce

EROS9: Wilderness \& other roadless areas

EROS10: Undeveloped areas near roads

EROS11: Partially developed, roaded areas

EROS12: Intensively developed winter sports sites

$\begin{array}{rrrrr}0.72 & 24.96 & 30.66 & 1.86 & 8.00 \\ 4.09 & 17.34 & 22.67 & 4.01 & 8.16 \\ 7.27 & 17.35 & 24.09 & 8.34 & 11.20 \\ 17.08 & 25.94 & 27.29 & 13.87 & 18.29\end{array}$

4.32

11.26

8.08

15.30

0.62

4.80

7.11

10.68

\subsection{4}

9.68

20.16

28.88

23.95
16.48
24.31
22.42

\subsection{1}

21.79

16.62

11.22

26.34

19.95

27.61

17.24
8.00

8.16

18.29

4.88

13.40

10.93

15.45

\footnotetext{
IThe EROS index is a measure of the relative availability of recreation opportunities to households in different locations and is calculated separately for each of the 12 recreation environments.

Source: Outdoor Recreation and Wilderness Assessment Research, USDA Forest Service, Athens, GA.
}

almost non-existent among the eastern regions compared to opportunities in the two western regions. The Rocky Mountain region provides the greatest opportunities for all of the snow and ice-based environments except "developed winter sports sites" (EROS 12). For this recreation environment, the Pacific region ranks highest. The lack of these types of opportunity is understandable for the South region because of the lack of sufficient snowfall. However, the relative lack of effective opportunities in the North region, compared to the two western regions, indicates the presence of greater population competing for fewer available resources.

\section{Substitute Recreation Opportunities}

Availability of substitute recreation opportunities is important in modeling recreation demand and consumption. One general measure of the availability of substitutes for a recreation environment is provided by the average of the EROS indices for other recreation settings that might provide a reasonable substitute (table 13). Snow and ice environments were not considered as substitutes for land and water settings; but, land and water resources were regarded as potential substitutes for each other. Although substitute recreation opportunities can be expected to change in the future, along with recreation resource opportunities, the decision was made to hold the substitute indices constant at the base year level. Recreation supply and demand projections continued under the scenario of no change in the amount of substitutes available.

For all 12 environments, the largest substitute index values are found in the Rocky Mountain region. The most pronounced differences are between the eastern and western regions, because the large amounts of public lands in the West affect virtually all substitute index values. The North region ranks slightly below the South for all land environments, and for three of four water environments.

\section{Regional EROS Projections}

A relatively higher percentage of population growth to the year 2040 is expected to occur in the Pacific Coast and Rocky Mountain regions, as shown in table 8 . Although overall population, and thus demand, for recreation resources will continue to be greater in the eastern regions, the rate of change in demand likely will be greater in the western regions. National decreases in many, but not all, land, water, and snow/ice resources and environments will coincide with these increases in population, if recent trends continue as identified in 
Table 13.-Mean substitute effective recreation opportunity sef (SUBEROS) indices, by region. ${ }^{1}$

Land

SUBEROS 1:

SUBEROS2:

SUBEROS3:

SUBEROS4:

Water

SUBEROS5:

SUBEROS6:

SUBEROS7:

SUBEROS8:

Snow and lce

SUBEROSP:

SUBEROSIO:

SUBEROS1 1:

SUBEROS12:

\begin{abstract}
Wilderness and other extensive roadless areas
Undeveloped areas near roads

Partially developed, roaded areas

Intensively developed sites
\end{abstract}

9.155
8.704
8.248
6.827

16.913
15.169
15.286
13.908

9.100

Wild \& remote lakes/streams

Lakes/streams near roads

Lake/stream sites adjoined by roads

Intensively developed water sites

7.718

8.151

7.123

17.167
16.241
14.825
13.492

Wilderness \& other roadless areas

Undeveloped areas near roads

Partially developed, roaded areas

Intensively developed winter sports sites
7.221

5.995
5.434
4.047

19.923
15.886
14.300

13.986
17.572
15.644
15.507
14.950

18.222

16.452

16.944

16.899

20.683

17.145

15.715

17.878
9.483

9.130

8.448

7.671

9.510

7.630

8.349

7.705

11.293

10.530

10.093

9.047

11.406

9.822

10.199

9.437

\footnotetext{
ISUBEROS indices are means of the Effective Recreation Opportunity Set (EROS) indices of other resource categories that could substitute for the principal environment in which a recreational activity occurs. That is, the amount of effective opportunity in all other land and water resource categories is assumed to be a potential substitute setting for the land or water resource category in which the recreational activity predominantly occurs. See Cordell, English and Bergstrom (1989) or English and Cordell (1993) for details on the EROS indices.
}

table 4. The impact of these changes on regional effective recreation opportunities was examined in this study on a regional basis.

In the case of land-based environments, the results show effective recreation opportunities decreasing for "wilderness and remote back-country" (EROS 1), "extensive undeveloped areas near roads" (EROS 2), and "roaded and partially developed areas" (EROS 3) in all four RPA regions (table 14). The reductions range from $10 \%$ to $36 \%$ less in 2040 than in the 1987 base year, and are projected to be greatest in the North and South regions. In most cases, these decreases are two to three times greater than those in the western regions. One exception is for opportunities in "extensive undeveloped areas near roads" (EROS 2), where the Pacific Coast region falls just behind the North and South regions. Effective opportunities in "developed sites" (EROS 4) are projected to increase over time, although this increase should be much less for the South region than the other three regions. In fact, initial projections are expected to actually decrease for this region before gradually increasing. The greatest increase in opportunities should occur in the Pacific Coast Region followed by the Rocky Mountains and North regions.

The projected effective opportunity changes in water-based environments show varied results. In the case of "Wild and Scenic or other remote lakes and streams" (EROS5), effective opportunities are expected to decrease in the eastern regions, and increase in the western regions. Although these changes are relatively small (i.e., averaging $1 \%$ to $4.5 \%$ ) when compared with other environments, the results are interesting given the specific regional population changes expected and the fact that resources available are expected to increase for this category. Opportunities associated with "lakes or streams near roads" (EROS 6) are expected to decrease for all four RPA regions, again with much greater decreases in the North and South regions than in the western regions. Conversely, effective opportunities in "partially developed lakes or streams with roads or crossings" (EROS 7) and "developed water sites" (EROS 8) are expected to increase over time for all four RPA regions. Opportunities in the South region are expected to increase at a slower rate than is the case for the other three regions.

Projected opportunity changes for snow and icebased environments are expected to decrease for all environments except "developed winter sports sites" (EROS 12). The decreases in "wilderness and other remote back-country" (EROS 9) and "extensive undeveloped areas near roads" (EROS 10) should be much greater in the South region. The Pacific Coast region is expected to show slower rates of decreases in "roaded 
Percent change from 1987

Resources and environments
(region)

\section{0}

2010

2020

2030

2040

Land

EROS 1: Wilderness and other extensive roadless areas

$\begin{array}{ll}\text { North } & -9.3 \\ \text { Pacific Coast } & -3.8 \\ \text { Rocky Mountains } & -2.8 \\ \text { South } & -9.9 \\ \text { Undeveloped areas near roads } & \\ \text { North } & -8.4 \\ \text { Pacific Coast } & -5.6 \\ \text { Rocky Mountains } & -3.1 \\ \text { South } & -8.9\end{array}$

-9.3
-3.8
-2.8
-9.9

-8.4
-5.6
-3.1
-8.9

\section{$-17.0$}

$-6.3$

$-4.7$

$-16.1$

EROS 2:

EROS 3: $\begin{aligned} & \text { Partially developed, roaded areas } \\ & \text { North }\end{aligned}$

Pacific Coast $\quad-5.2$

Rocky Mountains

South

-5.2
-4.9

$-13.3$

EROS 4:

Intensively developed sites

North

Pacific Coast

Rocky Mountains

South

\section{4}

4.9

3.8

$-1.6$

Water

EROS 5: Wild and remote lakes/streams

North

Pacific Coast

Rocky Mountains

South

EROS 6: Lakes/streams near roads

North

Pacific Coast

Rocky Mountains

South

EROS 7: Lake/stream sites adjoined

North

Pacific Coast

Rocky Mountains

South

Intensively developed water sites

North

Pacific Coast

Rocky Mountains

South

EROS 8:

-0.2
0.7
0.2
-2.7
-5.9
-2.7
-1.6
-10.8

by roads

ads

2.6

2.7

$-1.4$

\section{4}

6.3

5.7

0.6
$-15.3$

$-9.5$

$-5.6$

$-14.0$

$-17.7$

$-8.9$

$-8.3$

$-20.8$

\section{5}

9.2

6.9

$-0.9$

\section{$-0.7$}

1.4

0.4

$-3.5$

$-10.3$

$-3.6$

$-2.3$

$-15.6$

2.8

4.9

4.9

$-0.9$

5.7

12.0

10.3

2.9

Snow and lce

EROS 9: $\quad$ Wilderness \& other roadless areas

North

Pacific Coast

Rocky Mountains

South

$-3.7$

$-2.3$

$-1.5$

$-14.4$

EROS 10:

Undeveloped areas near roads

North

Pacific Coast

$-4.8$

Rocky Mountains

South

$-3.6$

$-2.6$

$-12.4$

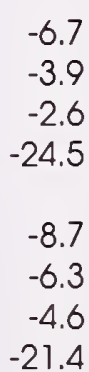

$-6.7$

$-2.6$

$-24.5$

$-8.7$

$-6.3$

$-21.4$
$-23.4$

$-9.2$

$-6.8$

$-22.7$

$-21.6$

$-14.1$

$-8.4$

$-19.4$

$-24.5$

$-12.6$

$-12.1$

$-27.8$

5.2

13.3

9.8

$-0.4$

$-1.2$

1.9

0.5

$-4.6$

$-14.5$

$-5.4$

$-3.4$

$-20.8$

4.0

7.2

7.0

$-0.4$

8.0

17.1

14.2

4.9

$-9.3$

$-5.5$

$-4.1$

$-30.9$

$-12.9$

$-9.7$

$-6.9$

$-30.0$

\section{$-27.4$}

$-11.8$

$-8.6$

$-26.0$

$-26.2$

$-18.6$

$-11.3$

$-23.1$

$-28.3$

$-16.3$

$-15.4$

$-31.8$

9.1

17.2

12.7

2.8

$-31.2$

$-14.6$

$-10.4$

$-30.5$

$-30.5$

$-22.6$

$-13.9$

$-26.9$

$-32.8$

$-19.9$

$-18.8$

$-36.2$

12.9

21.5

15.6

5.8

$\begin{array}{rr}-1.0 & -1.0 \\ 2.2 & 2.4 \\ 0.6 & 0.6 \\ -4.5 & -4.8 \\ -16.1 & \\ -7.1 & -18.4 \\ -4.5 & -9.0 \\ -23.0 & -5.6 \\ & -25.5 \\ 7.2 & \\ 9.2 & 10.1 \\ 9.0 & 11.4 \\ 2.1 & 11.3 \\ & 4.5 \\ 12.4 & \\ 22.6 & 16.3 \\ 18.3 & 28.1 \\ 9.6 & 22.2 \\ & 13.6\end{array}$

$-1.0$

2.4

0.6

$-18.4$

$-9.0$

$-5.6$

$-25.5$

10.1

11.4

4.5

16.3

28. 1

13.6

-14.1
-8.7
-6.7
-39.6
-19.6
-16.1
-11.5
-42.0

(Continued) 
Percent change from 1987

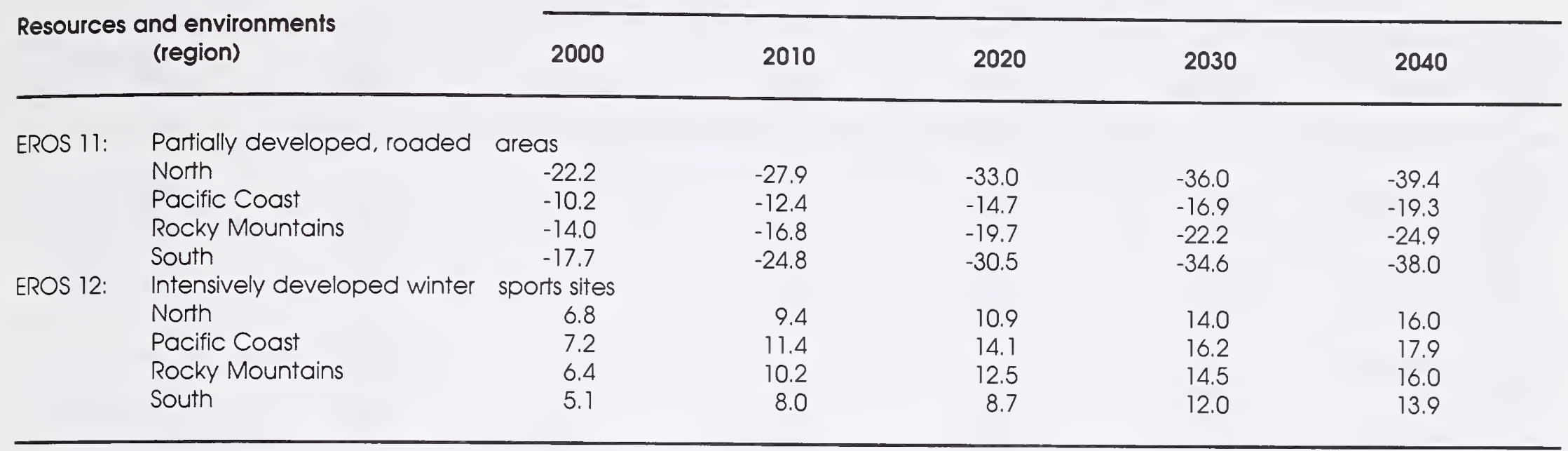

Source: Outdoor Recreation and Wilderness Assessment Research, USDA Forest Service, Athens, GA.

and partially developed areas" (EROS11) than the other three regions. In the case of "developed winter sports sites" (EROS 12), all four RPA regions are expected to increase at similar rates.

\section{RECREATION TRIPS}

\section{Current Consumption}

Because the number of trips consumed is closely tied to population, more trips were consumed in the North region in 1987 than in any other region for all activities except motor-boating (table 15). For motor-boating, the South region consumed the most recreation trips. Because of its much smaller population base, the Rocky Mountain region accounts for the smallest proportion of trips consumed.

Across the four regions, the same seven activities were the most popular, although the rankings within regions varied (table 16). For all four regions, driving for pleasure ranked at the top. For three of the regions, sightseeing ranked second. In the Rocky Mountain region, picnicking was the second most popular activity. In the eastern two regions, outdoor pool swimming ranked seventh;but, in the western regions, it ranked either third or fourth.

\section{Projected Supply}

Driving for pleasure and sightseeing are expected to remain the top two activities through 2040, in terms of the nationwide volume of expected supply of annual trips (table 15). Neither activity ranks lower than fourth in any RPA region. Closely related activities that are often consumed together, sightseeing and driving for pleasure are both dependent on roads and motorized transportation.

There is greater variety among the regions in the activities which are expected to show the greatest percentage increases in expected supply or trip consumption by 2040 (table 17). Five activities are among the top 10 fastest growing activities in all regions. These include downhill skiing, sailing, rafting/tubing, running/jogging, and outdoor pool swimming. The RPA projections for downhill skiing, however, have met sharp criticism from more than one reviewer. Although an extension of recent past trends projects continued steady growth in the developed winter sports sites recreation environment, in reality, the number of downhill skiing resorts has declined in recent years. While larger resorts have been steadily increasing their skier capacity, the overall trend has been a reduction in skier capacity and a gradual decline in the annual number of skier visits (United Ski Industries Association 1991).

It also should be noted that the high projected growth rates of sailing and rafting/tubing are largely a function of their small number of base year trips, making moderate growth in trips appear deceptively large in terms of percent change. Moreover, the regression models for expected supply of sailing and rafting/tubing trips were among the least stable of all of the activity models. The sailing model explained $33 \%$ of the variation in sailing trips and the rafting/tubing model performed worst of all, with an adjusted R-square of 0.12 . 
Table 15.-Current consumption of recreation trips away from home and indices of future growth to 2040, if recent past trends in resource availability continue.

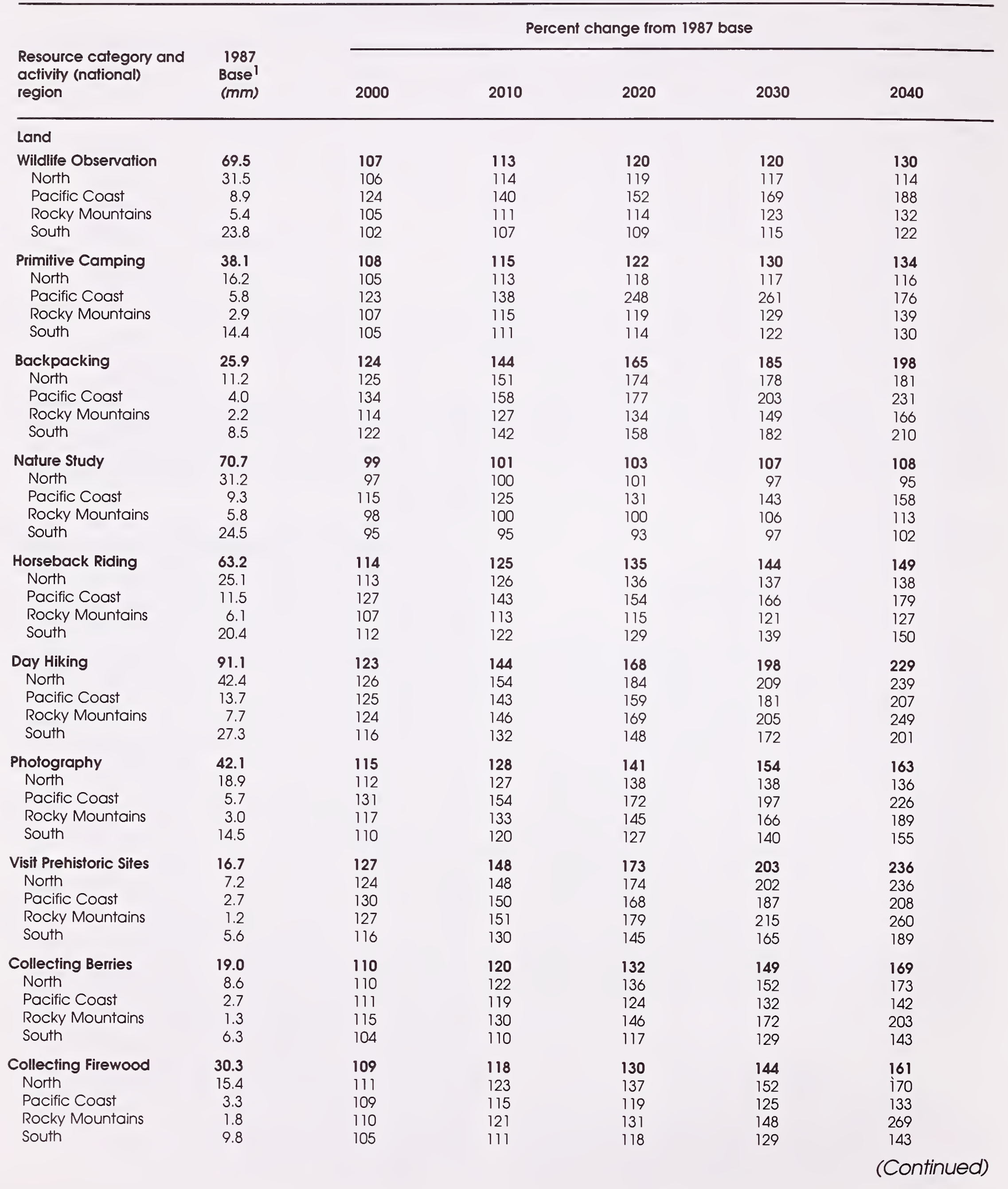


Table 15.-(continued).

Percent change from 1987 base

Resource category and
activity (national)
region

1987

Base ${ }^{1}$

(mm)
2000

\section{6}

111

147

118

111

Rocky Mountains

South

\section{Running and Jogging}

North

Pacific Coast

Rocky Mountain

South

\section{Bicycling}

North

Pacific Coast

Rocky Mountain

South

Offroad Vehicle Driving

North

Pacific Coast

Rocky Mountains

South

83.7

38.4

13.0

6.3

26.0

114.5

47.5

18.9

10.3

37.8

80.2

35.9

11.0

5.9

27.4

\section{Visiting Museums}

North

Pacific Coast

Rocky Mountains

South

9.7

4.7

1.2

0.6

3.2

\section{Attend Special Events}

North

Pacific Coast

Rocky Mountains

South

73.7

35.2

9.8

5.2

23.6

73.0

Visiting Historic Sites

North

Pacific Coast

Rocky Mountains

South

33.9

10.2

4.9

24.0

Driving For Pleasure $\quad \mathbf{4 2 1 . 4}$

North

Pacific Coast

Rocky Mountains

South

198.3

52.5

27.9

142.7

\section{Family Gatherings}

North

Pacific Coast

Rocky Mountains

South

74.4

35.3

11.4

5.9

21.7

292.1

Sightseeing

North

Pacific Coast

Rocky Mountains

South
128.2

45.1

19.8

99.0

\section{1}

125

169

134

124

124

118

157

126

117

104

100

124

103

100

118

113

148

119

112

115

110

144

116

110

117

118

121

122

110

110

107

132

111

105

121

117

150

120

115

114

115

122

118

108
132

125

189

136

124

160

152

241

169

149

\section{6}

140

210

153

136

\section{8}

104

138

106

103

134

129

192

139

125

129

122

182

133

122

133

138

137

143

121

120

117

155

122

112

139

136

192

138

130

128

131

137

134

117
148

136

230

151

134

192

177

317

201

170

170

158

264

176

150

112

106

148

109

104

\section{2}

142

235

155

135

\section{4}

133

218

146

131

152

159

151

166

133

129

125

273

129

116

160

153

232

154

143

144

148

149

151

126

2030

2040

183

140

361

208

174

260

189

591

317

248

218

165

445

259

206

121

105

171

121 
Table 15.-(continued).

Percent change from 1987 base

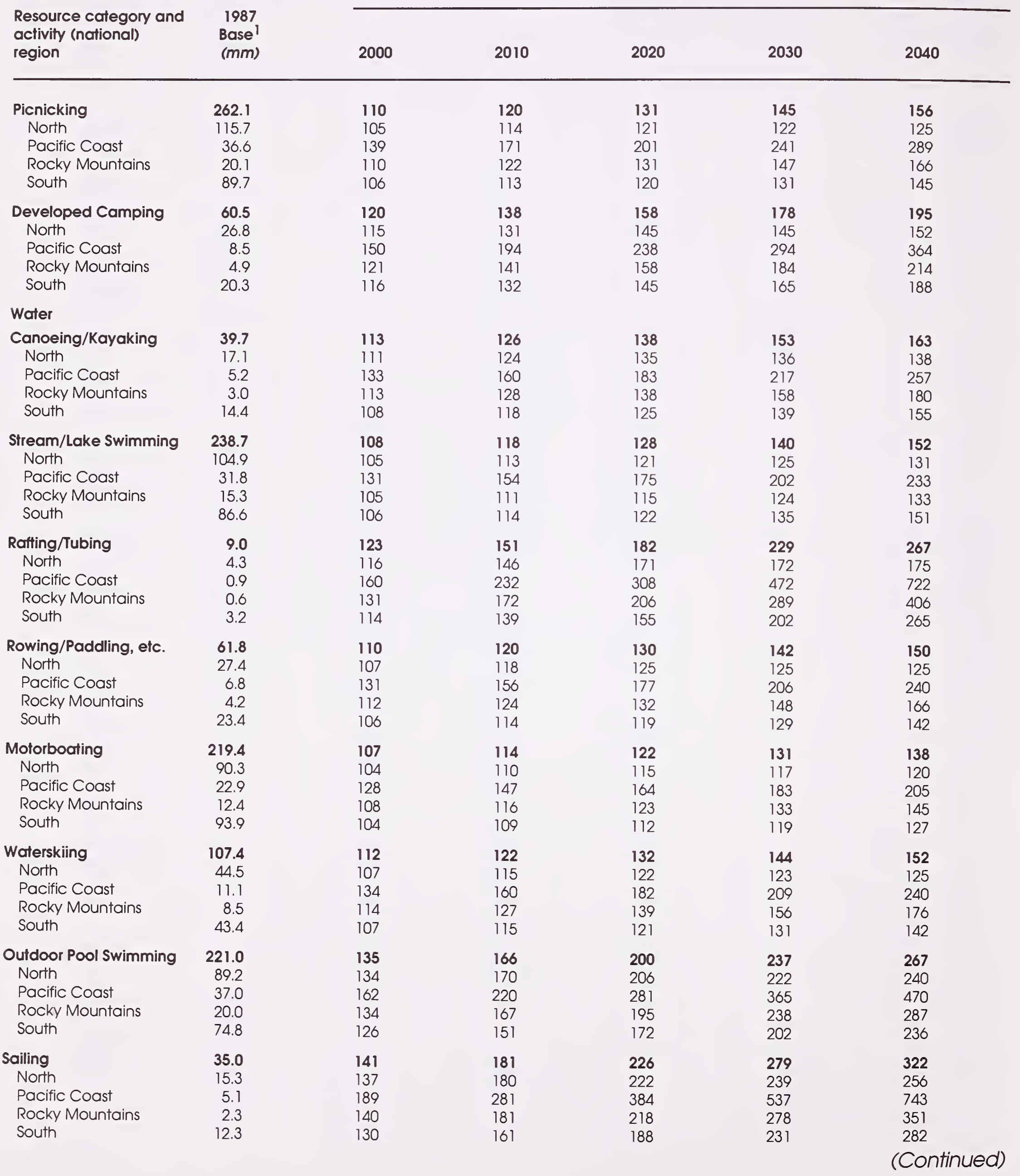


Table 15.-(continued).

Percent change from 1987 base

\begin{tabular}{|c|c|c|c|c|c|c|}
\hline \multirow{2}{*}{$\begin{array}{l}\text { Resource category and } \\
\text { activity (national) } \\
\text { region }\end{array}$} & \multirow{2}{*}{$\begin{array}{l}1987 \\
\text { Base }^{1} \\
(\mathrm{~mm})\end{array}$} & \\
\hline & & 2000 & 2010 & 2020 & 2030 & 2040 \\
\hline \multicolumn{7}{|l|}{ Snow and lce } \\
\hline $\begin{array}{l}\text { Cross-Country Skiing } \\
\text { North } \\
\text { Pacific Coast } \\
\text { Rocky Mountains } \\
\text { South }\end{array}$ & $\begin{array}{l}9.7 \\
5.3 \\
1.3 \\
1.2 \\
2.0\end{array}$ & $\begin{array}{l}125 \\
112 \\
154 \\
132 \\
126\end{array}$ & $\begin{array}{l}136 \\
120 \\
191 \\
155 \\
143\end{array}$ & $\begin{array}{l}142 \\
116 \\
211 \\
161 \\
146\end{array}$ & $\begin{array}{r}141 \\
88 \\
245 \\
181 \\
157\end{array}$ & $\begin{array}{r}126 \\
66 \\
277 \\
197 \\
165\end{array}$ \\
\hline $\begin{array}{l}\text { Downhill Skiing } \\
\text { North } \\
\text { Pacific Coast } \\
\text { Rocky Mountains } \\
\text { South }\end{array}$ & $\begin{array}{r}64.3 \\
32.7 \\
11.2 \\
8.9 \\
11.5\end{array}$ & $\begin{array}{l}160 \\
174 \\
178 \\
168 \\
136\end{array}$ & $\begin{array}{l}207 \\
243 \\
241 \\
230 \\
163\end{array}$ & $\begin{array}{l}256 \\
309 \\
295 \\
290 \\
184\end{array}$ & $\begin{array}{l}305 \\
336 \\
364 \\
376 \\
213\end{array}$ & $\begin{array}{l}338 \\
357 \\
436 \\
478 \\
243\end{array}$ \\
\hline $\begin{array}{l}\text { Snowmobiling } \\
\text { North } \\
\text { Pacific Coast } \\
\text { Rocky Mountains } \\
\text { South }\end{array}$ & $\begin{array}{l}17.7 \\
11.0 \\
2.1 \\
1.6 \\
3.1\end{array}$ & $\begin{array}{l}120 \\
123 \\
137 \\
124 \\
110\end{array}$ & $\begin{array}{l}131 \\
140 \\
162 \\
141 \\
117\end{array}$ & $\begin{array}{l}137 \\
146 \\
179 \\
148 \\
117\end{array}$ & $\begin{array}{l}141 \\
135 \\
204 \\
162 \\
120\end{array}$ & $\begin{array}{l}137 \\
124 \\
228 \\
174 \\
122\end{array}$ \\
\hline
\end{tabular}

'Regional trips may not sum exactly to the national total because of rounding.

Source: Outdoor Recreation and Wilderness Assessment Research, USDA Forest Service, Athens, GA.

Nonetheless, given the uniform methods that were applied to each of the activity models and projections, downhill skiing ranked first in the North and Rocky Mountains, while sailing was projected to grow fastest in the Pacific Coast and South. Sailing is also highly ranked in the North and Rocky Mountains. Rafting/ tubing is expected to be the second fastest growing activity in every region but the North, where it ranks 10th

Table 16.-Most popular outdoor recreation activities in volume of trips consumed by region, 1987.

\begin{tabular}{lcccc}
\hline & \multicolumn{5}{c}{ Region } \\
\cline { 2 - 5 } Activity & $\begin{array}{c}\text { Pacific } \\
\text { North }\end{array}$ & $\begin{array}{c}\text { Rocky } \\
\text { Coast }\end{array}$ & Mountains & South \\
\hline & \multicolumn{5}{c}{ (rank) } & \\
Driving for pleasure & 1 & 1 & 1 & 1 \\
Sightseeing & 2 & 2 & 4 & 2 \\
Walking for pleasure & 3 & 3 & 5 & 5 \\
Picnicking & 4 & 5 & 2 & 4 \\
Non-pool swimming & 5 & 6 & 6 & 6 \\
Motorboating & 6 & 7 & 7 & 3 \\
Pool swimming & 7 & 4 & 3 & 7 \\
& \multicolumn{5}{c}{} & & & \\
\hline
\end{tabular}

Source: Outdoor Recreation and Wilderness Assessment Research, USDA Forest Service, Athens, GA.
Growth rates in trip consumption also vary by region, and reflect projected growth in regional population. All of the 10 fastest growing activities in the Pacific Coast region are expected to show increases of more than $200 \%$ by 2040. Table 17.-Top 10 fastest growing activities in trip consumption to 2040 , as percentage of 1987 base year trips, by region.

\begin{tabular}{|c|c|c|c|c|}
\hline \multirow[b]{2}{*}{ Activity } & \multicolumn{4}{|c|}{ Region } \\
\hline & $\begin{array}{l}\text { Pacific } \\
\text { North }\end{array}$ & $\begin{array}{l}\text { Rocky } \\
\text { Coast }\end{array}$ & Mountains & South \\
\hline & \multicolumn{4}{|c|}{ (rank) } \\
\hline Downhill skiing & 1 & 6 & 1 & 4 \\
\hline Sailing & 2 & 1 & 3 & 1 \\
\hline Outdoor pool swimming & 3 & 4 & 5 & 5 \\
\hline Day hiking & 4 & - & 9 & 8 \\
\hline Visiting prehistoric sites & 5 & - & 7 & 9 \\
\hline Visiting historic sites & 6 & - & 10 & - \\
\hline Running/jogging & 7 & 3 & 4 & 3 \\
\hline Sightseeing & 8 & - & - & - \\
\hline Backpacking & 9 & - & - & 6 \\
\hline Rafting/tubing & 10 & 2 & 2 & 2 \\
\hline Family gatherings & - & 10 & - & - \\
\hline Collecting firewood & - & - & 6 & - \\
\hline Bicycling & - & 5 & 8 & 7 \\
\hline Visiting museums & - & 7 & - & - \\
\hline Developed camping & - & 8 & - & 10 \\
\hline Walking for pleasure & - & 9 & - & - \\
\hline
\end{tabular}

Source: Outdoor Recreation and Wilderness Assessment Research, USDA Forest Service, Athens, GA. 
Nine of these are expected to grow by more than $250 \%$. In the Rocky Mountain region, 10 activities are expected to double trip consumption by 2040. Eight of these are projected to increase by more than $150 \%$. By contrast, the top two activities in both the North and South regions are projected to grow as much as $150 \%$ by 2040 .

Three activities-bicycling, day hiking, and visiting prehistoric sites-rank among the top 10 consumption growth activities in three of four regions. Only in the North region is sightseeing expected to grow rapidly. Collecting firewood is expected to grow relatively faster only in the Rocky Mountain region. The Pacific Coast is the only region where visiting museums and walking for pleasure are among the fastest growing activities. Some shifting of the order of activities in terms of the expected supply in absolute number of trips is expected across the four RPA regions; but, generally the same group of activities should remain at the top. Pool swimming trips are projected to be the most popular in both the Pacific Coast and Rocky Mountain regions by 2040. In the North, driving for pleasure and sightseeing are expected to still rank first and second, respectively, in number of trips consumed. In the South, driving for pleasure is expected to remain the most popular activity; but, pool swimming will replace sightseeing as the second most frequently consumed activity.

\section{Projected Demand}

Percentage growth in maximum preferred demand (MPD) for recreation trips, paced by changes in income, age, and population is expected to be greater in the Rocky Mountains and Pacific Coast regions than in the eastern regions, for almost every activity (table 18). For day hiking, downhill skiing, visiting prehistoric sites, rafting/tubing, sightseeing, and visiting historic sites, percentage growth in trips demanded is expected to be greater in the Rocky Mountain region than in the Pacific Coast region. For all other activities, the reverse is true.

Again, there are regional differences in the ranking of activities for which demand is expected to grow fastest over the next 50 years (table 19). Four activities rank among the highest 10 percentage growth activities for all regions. These activities are downhill skiing, sailing, running/jogging, and backpacking. Three different activities rank at the top for the four regions. Downhill skiing is expected to have the greatest growth in the North, rafting/tubing in the Rocky Mountains, and sailing in the Pacific Coast and South.
There also are regional differences in the overall rate of growth in MPD. In the North, seven activities have MPD indices at or above 200 by the year 2040. Nine activities reach that level in the South. In the western regions, all of the 10 fastest growing activities have MPD indices greater than 300 by 2040 . Three activities in the Rocky Mountains and seven in the Pacific Coast have MPD indices greater than 400 .

These changes primarily reflect the expected higher percentage growth in population in the western regions. From 1987 to 2040, the projected growth by region is: Pacific Coast 46.9\%; Rocky Mountains 34.5\%; South $24.9 \%$; and North $14.2 \%$, as shown in table 8 . Projections of percentage growth of the percent of households with at least $\$ 30,000$ real income also shows significant gains in all regions. The South region leads the way with a projected $54.6 \%$ increase, followed by the Rocky Mountains (54.0\%), Pacific Coast (46.1\%) and North (33.1\%). The combination of population and income projections explain why the North region trails in projected demand for most of the recreation activities.

\section{COMPARISON OF DEMAND AND SUPPLY}

Maximum preferred demand and expected supply are closely related concepts. Both measure consumption of recreation trips, but under different assumptions. MPD measures consumption with price held constant and no constraints on the availability of recreation facilities and resources. Expected supply measures the number of trips that households would actually produce and consume at varying price levels and assumed futures for the availability of resources. If expected supply exceeds MPD, no gap is present. However, if projected MPD exceeds expected supply, there is a shortage or "gap."' A gap implies that the number of trips households would like to produce, if prices were unchanged, surpasses the number of trips that they would actually produce.

Table 20 lists the MPD, expected supply, and resultant gaps for each of the activities, by region and for the nation as a whole. For a number of activities, gaps appeared in some regions, but not in others. For example, the North region should have an adequate supply of canoe trip opportunities through the year 2040; but, the other three regions show gaps in supply, as much as $61 \%$ of current trips in the Pacific Coast region. 
Table 18.-Maximum preferred demand for recreation trips away from home and indices of future growth to 2040.1

Future number of trips as percentage of 1987 demand

Resource category and
activity (national)
region

1987

Trips $^{2}$

(mm)

2000

2010

2020

2030

2040

Land

Wildlife Observation

North

Pacific Coast

Rocky Mountains

South

\section{Primitive Camping}

North

Pacific Coast

Rocky Mountains

South

\section{Backpacking}

North

Pacific Coast

Rocky Mountains

South

Nature Study

North

Pacific Coast

Rocky Mountains

South

\section{Horseback Riding}

North

Pacific Coast

Rocky Mountains

South

\section{Day Hiking}

North

Pacific Coast

Rocky Mountains

South

Photography

North

Pacific Coast

Rocky Mountains

South

69.5

31.5

8.9

5.4

23.8

38.1

16.2

5.8

2.9

14.4

25.9

11.2

4.0

2.2

8.5

70.7

31.2

9.3

5.8

24.5

63.2

25.1

11.5

6.1

20.4

91.1

42.4

13.7

7.7

27.3

42.1

18.9

5.7

3.0

14.5

Visit Prehistoric Sites

North

Pacific Coast

Rocky Mountains

South

\section{Collecting Berries}

North

Pacific Coast

Rocky Mountains

South

16.7

7.2

2.7

1.2

5.6

19.0

8.6

2.7

1.3

6.3

30.3

15.4

3.3

1.8

9.8
116

112

143

118

111

114

110

143

115

109

134

129

167

136

126

105

101

129

107

100

123

118

150

123

117

131

132

142

138

122

123

118

154

125

116

133

130

139

134

120

\section{3}

114

119

117

106

113

113

116

116

106
131

127

179

136

122

127

122

178

130

118

164

160

231

172

151

113

108

153

117

105

141

135

190

142

132

161

167

177

178

145

143

139

202

150

133

160

159

167

165

138

126

130

132

135

114

127

127

128

131

114
146

146
138

211

150

130

140

131

208

142

125

196

187

295

203

171

120

113

172

124

108

160

150

226

158

144

198

206

212

221

168

165

156

248

171

145

192

193

193

201

158

143

148

143

154

124

143

143

137

148

122

162

$139 \quad 140$

$256 \quad 308$

$173 \quad 201$

$145 \quad 162$

154

$131 \quad 132$

$246 \quad 290$

$161 \quad 183$

$137 \quad 151$

$230 \quad 255$

$193 \quad 197$

$385 \quad 497$

$252 \quad 309$

$203 \quad 238$

$131 \quad 138$

$111 \quad 111$

$202 \quad 238$

$\begin{array}{ll}140 & 158\end{array}$

$117 \quad 128$

$177 \quad 190$

$153 \quad 156$

$267 \quad 312$

$180 \quad 203$

$159 \quad 176$

$244 \quad 293$ 
Table 18.-(continued).

Future number of trips as percentage of 1987 demand

\begin{tabular}{|c|c|c|c|c|c|c|}
\hline \multirow{2}{*}{$\begin{array}{l}\text { Resource category and } \\
\text { activity (national) } \\
\text { region }\end{array}$} & \multirow{2}{*}{$\begin{array}{l}1987 \\
\text { Trips }^{2} \\
(\mathrm{~mm})\end{array}$} & & & & & \\
\hline & & 2000 & 2010 & 2020 & 2030 & 2040 \\
\hline Walking for Pleasure & 266.4 & 116 & 131 & 146 & 164 & 177 \\
\hline North & 118.0 & 111 & 125 & 137 & 137 & 137 \\
\hline Pacific Coast & 40.3 & 147 & 187 & 224 & 275 & 337 \\
\hline Rocky Mountains & 18.8 & 118 & 136 & 150 & 176 & 206 \\
\hline South & 89.3 & 109 & 121 & 128 & 144 & 162 \\
\hline Running and Jogging & 83.7 & 133 & 163 & 197 & 234 & 262 \\
\hline North & 38.4 & 126 & 157 & 184 & 187 & 191 \\
\hline Pacific Coast & 13.0 & 171 & 241 & 313 & 420 & 560 \\
\hline Rocky Mountains & 6.3 & 136 & 173 & 206 & 261 & 329 \\
\hline South & 26.0 & 124 & 148 & 167 & 201 & 241 \\
\hline Bicycling & 114.5 & 125 & 148 & 173 & 202 & 222 \\
\hline North & 47.5 & 120 & 143 & 163 & 165 & 167 \\
\hline Pacific Coast & 18.9 & 160 & 217 & 272 & 354 & 457 \\
\hline Rocky Mountains & 10.3 & 128 & 157 & 181 & 223 & 274 \\
\hline South & 37.8 & 117 & 136 & 150 & 176 & 206 \\
\hline Offroad Vehicle Driving & 80.2 & 105 & 111 & 118 & 125 & 130 \\
\hline North & 35.9 & 101 & 106 & 109 & 108 & 108 \\
\hline Pacific Coast & 11.0 & 132 & 154 & 171 & 191 & 213 \\
\hline Rocky Mountains & 5.9 & 105 & 111 & 115 & 124 & 133 \\
\hline South & 27.4 & 101 & 104 & 106 & 111 & 118 \\
\hline Visiting Museums & 9.7 & 118 & 136 & 153 & 174 & 188 \\
\hline North & 4.7 & 113 & 130 & 144 & 145 & 146 \\
\hline Pacific Coast & 1.2 & 149 & 192 & 232 & 289 & 359 \\
\hline Rocky Mountains & 0.6 & 121 & 143 & 160 & 190 & 226 \\
\hline South & 3.2 & 112 & 125 & 135 & 153 & 174 \\
\hline Attend Special Events & 73.7 & 114 & 127 & 141 & 157 & 168 \\
\hline North & 35.2 & 109 & 122 & 132 & 132 & 133 \\
\hline Pacific Coast & 9.8 & 143 & 179 & 211 & 255 & 308 \\
\hline Rocky Mountains & 5.2 & 116 & 132 & 144 & 167 & 193 \\
\hline South & 23.6 & 108 & 118 & 125 & 139 & 155 \\
\hline Visiting Historic Sites & 73.0 & 122 & 143 & 169 & 203 & 241 \\
\hline North & 33.9 & 122 & 147 & 175 & 203 & 238 \\
\hline Pacific Coast & 10.2 & 130 & 154 & 177 & 208 & 247 \\
\hline Rocky Mountains & 4.9 & 128 & 157 & 189 & 240 & 306 \\
\hline South & 24.0 & 113 & 128 & 143 & 167 & 198 \\
\hline Driving for Pleasure & 421.4 & 115 & 128 & 142 & 157 & 167 \\
\hline North & 198.3 & 110 & 122 & 132 & 133 & 133 \\
\hline Pacific Coast & 52.5 & 145 & 181 & 213 & 253 & 301 \\
\hline Rocky Mountains & 27.9 & 116 & 131 & 143 & 163 & 186 \\
\hline South & 142.7 & 109 & 119 & 126 & 139 & 153 \\
\hline Family Gatherings & 74.4 & 119 & 135 & 152 & 170 & 182 \\
\hline North & 35.3 & 113 & 129 & 141 & 142 & 144 \\
\hline Pacific Coast & 11.4 & 154 & 197 & 238 & 288 & 346 \\
\hline Rocky Mountains & 5.9 & 120 & 138 & 153 & 176 & 203 \\
\hline South & 21.7 & 112 & 125 & 134 & 149 & 166 \\
\hline Sightseeing & 292.1 & 118 & 136 & 156 & 183 & 212 \\
\hline North & 128.2 & 118 & 138 & 160 & 183 & 211 \\
\hline Pacific Coast & 45.1 & 131 & 153 & 172 & 196 & 224 \\
\hline Rocky Mountains & 19.8 & 122 & 144 & 168 & 203 & 247 \\
\hline South & 99.0 & 111 & 122 & 134 & 152 & 174 \\
\hline
\end{tabular}

(Continued) 
Table 18.-(continued).

Future number of trips as percentage of 1987 demand

\begin{tabular}{|c|c|c|c|c|c|c|}
\hline $\begin{array}{l}\text { Resource category and } \\
\text { activity (national) } \\
\text { region }\end{array}$ & $\begin{array}{l}1987 \\
\text { Trips }^{2} \\
(\mathrm{~mm})\end{array}$ & 2000 & 2010 & 2020 & 2030 & 2040 \\
\hline $\begin{array}{l}\text { Picnicking } \\
\text { North } \\
\text { Pacific Coast } \\
\text { Rocky Mountains } \\
\text { South }\end{array}$ & $\begin{array}{r}262.1 \\
115.7 \\
36.6 \\
20.1 \\
89.7\end{array}$ & $\begin{array}{l}108 \\
103 \\
137 \\
109 \\
103\end{array}$ & $\begin{array}{l}117 \\
111 \\
166 \\
119 \\
109\end{array}$ & $\begin{array}{l}126 \\
116 \\
189 \\
126 \\
112\end{array}$ & $\begin{array}{l}136 \\
116 \\
219 \\
140 \\
121\end{array}$ & $\begin{array}{l}144 \\
115 \\
254 \\
156 \\
131\end{array}$ \\
\hline $\begin{array}{l}\text { Developed Camping } \\
\text { North } \\
\text { Pacific Coast } \\
\text { Rocky Mountains } \\
\text { South }\end{array}$ & $\begin{array}{r}60.5 \\
26.8 \\
8.5 \\
4.9 \\
20.3\end{array}$ & $\begin{array}{l}120 \\
115 \\
153 \\
121 \\
114\end{array}$ & $\begin{array}{l}137 \\
131 \\
197 \\
141 \\
127\end{array}$ & $\begin{array}{l}155 \\
145 \\
238 \\
157 \\
137\end{array}$ & $\begin{array}{l}173 \\
147 \\
290 \\
182 \\
153\end{array}$ & $\begin{array}{l}186 \\
149 \\
351 \\
210 \\
174\end{array}$ \\
\hline \multicolumn{7}{|l|}{ Water } \\
\hline $\begin{array}{l}\text { Canoeing/Kayaking } \\
\text { North } \\
\text { Pacific Coast } \\
\text { Rocky Mountains } \\
\text { South }\end{array}$ & $\begin{array}{r}39.7 \\
17.1 \\
5.2 \\
3.0 \\
14.4\end{array}$ & $\begin{array}{l}113 \\
109 \\
141 \\
116 \\
107\end{array}$ & $\begin{array}{l}126 \\
122 \\
177 \\
134 \\
117\end{array}$ & $\begin{array}{l}140 \\
132 \\
210 \\
147 \\
124\end{array}$ & $\begin{array}{l}157 \\
131 \\
258 \\
174 \\
139\end{array}$ & $\begin{array}{l}169 \\
131 \\
318 \\
205 \\
158\end{array}$ \\
\hline $\begin{array}{l}\text { Stream/Lake Swimming } \\
\text { North } \\
\text { Pacific Coast } \\
\text { Rocky Mountains } \\
\text { South }\end{array}$ & $\begin{array}{r}238.7 \\
104.9 \\
31.8 \\
15.3 \\
86.6\end{array}$ & $\begin{array}{l}105 \\
101 \\
131 \\
105 \\
100\end{array}$ & $\begin{array}{l}110 \\
105 \\
152 \\
111 \\
104\end{array}$ & $\begin{array}{l}117 \\
108 \\
169 \\
116 \\
105\end{array}$ & $\begin{array}{l}124 \\
107 \\
189 \\
125 \\
111\end{array}$ & $\begin{array}{l}129 \\
107 \\
212 \\
136 \\
118\end{array}$ \\
\hline $\begin{array}{l}\text { Rafting/Tubing } \\
\text { North } \\
\text { Pacific Coast } \\
\text { Rocky Mountains } \\
\text { South }\end{array}$ & $\begin{array}{l}9.0 \\
4.3 \\
0.9 \\
0.6 \\
3.2\end{array}$ & $\begin{array}{l}111 \\
106 \\
146 \\
132 \\
100\end{array}$ & $\begin{array}{l}136 \\
135 \\
210 \\
194 \\
119\end{array}$ & $\begin{array}{l}164 \\
156 \\
275 \\
245 \\
128\end{array}$ & $\begin{array}{l}215 \\
148 \\
442 \\
417 \\
176\end{array}$ & $\begin{array}{l}255 \\
145 \\
720 \\
724 \\
249\end{array}$ \\
\hline $\begin{array}{l}\text { Rowing/Paddling, etc. } \\
\text { North } \\
\text { Pacific Coast } \\
\text { Rocky Mountains } \\
\text { South }\end{array}$ & $\begin{array}{r}61.8 \\
27.4 \\
6.8 \\
4.2 \\
23.4\end{array}$ & $\begin{array}{l}112 \\
108 \\
136 \\
114 \\
107\end{array}$ & $\begin{array}{l}124 \\
120 \\
166 \\
129 \\
116\end{array}$ & $\begin{array}{l}136 \\
129 \\
192 \\
140 \\
122\end{array}$ & $\begin{array}{l}150 \\
129 \\
229 \\
160 \\
135\end{array}$ & $\begin{array}{l}159 \\
129 \\
273 \\
184 \\
150\end{array}$ \\
\hline $\begin{array}{l}\text { Motorboating } \\
\text { North } \\
\text { Pacific Coast } \\
\text { Rocky Mountains } \\
\text { South }\end{array}$ & $\begin{array}{r}219.4 \\
90.3 \\
22.9 \\
12.4 \\
93.9\end{array}$ & $\begin{array}{l}106 \\
102 \\
128 \\
106 \\
102\end{array}$ & $\begin{array}{l}111 \\
107 \\
147 \\
112 \\
105\end{array}$ & $\begin{array}{l}117 \\
110 \\
162 \\
116 \\
108\end{array}$ & $\begin{array}{l}123 \\
110 \\
178 \\
124 \\
113\end{array}$ & $\begin{array}{l}127 \\
110 \\
196 \\
132 \\
118\end{array}$ \\
\hline $\begin{array}{l}\text { Waterskiing } \\
\text { North } \\
\text { Pacific Coast } \\
\text { Rocky Mountains } \\
\text { South }\end{array}$ & $\begin{array}{r}107.4 \\
44.5 \\
11.1 \\
8.5 \\
43.4\end{array}$ & $\begin{array}{l}111 \\
107 \\
136 \\
112 \\
106\end{array}$ & $\begin{array}{l}121 \\
116 \\
163 \\
123 \\
114\end{array}$ & $\begin{array}{l}131 \\
123 \\
185 \\
131 \\
118\end{array}$ & $\begin{array}{l}141 \\
123 \\
211 \\
144 \\
127\end{array}$ & $\begin{array}{l}148 \\
124 \\
241 \\
159 \\
137\end{array}$ \\
\hline $\begin{array}{l}\text { Outdoor Pool Swimming } \\
\text { North } \\
\text { Pacific Coast } \\
\text { Rocky Mountains } \\
\text { South }\end{array}$ & $\begin{array}{r}221.0 \\
89.2 \\
37.0 \\
20.0 \\
74.8\end{array}$ & $\begin{array}{l}137 \\
132 \\
167 \\
141 \\
130\end{array}$ & $\begin{array}{l}169 \\
166 \\
231 \\
181 \\
157\end{array}$ & $\begin{array}{l}205 \\
196 \\
298 \\
217 \\
181\end{array}$ & $\begin{array}{l}242 \\
203 \\
389 \\
272 \\
215\end{array}$ & $\begin{array}{l}269 \\
208 \\
502 \\
337 \\
255\end{array}$ \\
\hline $\begin{array}{l}\text { Sailing } \\
\text { North } \\
\text { Pacific Coast } \\
\text { Rocky Mountains } \\
\text { South }\end{array}$ & $\begin{array}{r}35.0 \\
15.3 \\
5.1 \\
2.3 \\
12.3\end{array}$ & $\begin{array}{l}145 \\
137 \\
200 \\
150 \\
133\end{array}$ & $\begin{array}{l}188 \\
178 \\
307 \\
205 \\
168\end{array}$ & $\begin{array}{l}237 \\
218 \\
425 \\
256 \\
197\end{array}$ & $\begin{array}{l}293 \\
225 \\
603 \\
342 \\
244\end{array}$ & $\begin{array}{l}335 \\
232 \\
844 \\
453 \\
302\end{array}$ \\
\hline
\end{tabular}

(Continued) 
Table 18.-(continued).

Future number of trips as percentage of 1987 demand

\begin{tabular}{|c|c|c|c|c|c|c|}
\hline \multirow{2}{*}{$\begin{array}{l}\text { Resource category and } \\
\text { activity (national) } \\
\text { region }\end{array}$} & \multirow{2}{*}{$\begin{array}{l}1987 \\
\text { Trips } \\
(\mathrm{mm})\end{array}$} & \\
\hline & & 2000 & 2010 & 2020 & 2030 & 2040 \\
\hline \multicolumn{7}{|l|}{ Snow and lce } \\
\hline $\begin{array}{l}\text { Cross-Country Skiing } \\
\text { North } \\
\text { Pacific Coast } \\
\text { Rocky Mountains } \\
\text { South }\end{array}$ & $\begin{array}{l}9.7 \\
5.3 \\
1.3 \\
1.2 \\
2.0\end{array}$ & $\begin{array}{l}147 \\
140 \\
198 \\
157 \\
135\end{array}$ & $\begin{array}{l}177 \\
174 \\
288 \\
206 \\
160\end{array}$ & $\begin{array}{l}199 \\
190 \\
364 \\
232 \\
164\end{array}$ & $\begin{array}{l}212 \\
154 \\
484 \\
284 \\
181\end{array}$ & $\begin{array}{l}195 \\
121 \\
615 \\
336 \\
194\end{array}$ \\
\hline $\begin{array}{l}\text { Downhill Skiing } \\
\text { North } \\
\text { Pacific Coast } \\
\text { Rocky Mountains } \\
\text { South }\end{array}$ & $\begin{array}{r}64.3 \\
32.7 \\
11.2 \\
8.9 \\
11.5\end{array}$ & $\begin{array}{l}153 \\
164 \\
173 \\
168 \\
142\end{array}$ & $\begin{array}{l}197 \\
229 \\
234 \\
233 \\
176\end{array}$ & $\begin{array}{l}247 \\
294 \\
288 \\
298 \\
202\end{array}$ & $\begin{array}{l}298 \\
317 \\
361 \\
398 \\
241\end{array}$ & $\begin{array}{l}333 \\
336 \\
441 \\
521 \\
284\end{array}$ \\
\hline $\begin{array}{l}\text { Snowmobiling } \\
\text { North } \\
\text { Pacific Coast } \\
\text { Rocky Mountains } \\
\text { South }\end{array}$ & $\begin{array}{r}17.7 \\
11.0 \\
2.1 \\
1.6 \\
3.1\end{array}$ & $\begin{array}{l}119 \\
116 \\
132 \\
123 \\
114\end{array}$ & $\begin{array}{l}128 \\
128 \\
155 \\
139 \\
123\end{array}$ & $\begin{array}{l}134 \\
132 \\
170 \\
146 \\
123\end{array}$ & $\begin{array}{l}137 \\
118 \\
193 \\
161 \\
128\end{array}$ & $\begin{array}{l}130 \\
104 \\
215 \\
173 \\
131\end{array}$ \\
\hline
\end{tabular}

'Maximum preferred demand is the number of trips households would take if future per trip costs were unchanged from the base year amount and resource availability were unconstrained.

${ }^{2}$ Regional trips may not sum exactly to the national total because of rounding.

Source: Outdoor Recreation and Wilderness Assessment Research, USDA Forest Service, Athens, GA.

Table 19.-Top 10 fastest growing activities in maximum preferred demand to 2040 , as percentage of 1987 base, by region.

\begin{tabular}{|c|c|c|c|c|}
\hline Activity & $\begin{array}{c}\text { Region } \\
\text { Pacific } \\
\text { North }\end{array}$ & $\begin{array}{l}\text { Rocky } \\
\text { Coast }\end{array}$ & Mountains & South \\
\hline & \multicolumn{4}{|c|}{ (rank) } \\
\hline Downhill skiing & 1 & 7 & 2 & 2 \\
\hline Sailing & 4 & 1 & 3 & 1 \\
\hline Day hiking & 2 & - & 4 & 5 \\
\hline Rafting/tubing & - & 2 & 1 & 4 \\
\hline Pool swimming & 7 & - & 5 & 3 \\
\hline Visiting prehistoric sites & 3 & - & 8 & 8 \\
\hline Cross-country skiing & - & 3 & 6 & - \\
\hline Visiting historic sites & 6 & - & 10 & 10 \\
\hline Running/jogging & 10 & 4 & 7 & 6 \\
\hline Sightseeing & 5 & - & - & - \\
\hline Backpacking & 9 & 5 & 9 & 7 \\
\hline Bicycling & - & 6 & - & 9 \\
\hline Photography & - & 8 & - & - \\
\hline Collecting berries & 8 & - & - & - \\
\hline Visiting museums & - & 9 & - & - \\
\hline Developed camping & - & 10 & - & - \\
\hline
\end{tabular}

Source: Outdoor Recreation and Wilderness Assessment Research, USDA Forest Service, Athens, GA.
In most cases where no national gap was projected, regional gaps also were zero. Three exceptions were downhill skiing, snowmobiling, and rafting/tubing. Skiing had no projected gap in the North region, but projected gaps of 5\%,18\%, and 37\% in the Pacific Coast, Rocky Mountains and South regions, respectively. Snowmobiling showed a gap (9\%) of only in the South region. Rafting/tubing had an anticipated shortage of supply only in the Rocky Mountain region, where the gap was $318 \%$ of current trips. Because of the small number of base year trips ( 0.6 million), this rather large percentage gap translates to only 1.9 million trips.

There are some consistencies across regions regarding the five activities projected to have the highest percentage gaps (table 21). For all four regions, cross-country skiing is projected to have one of the five largest regional percentage gaps. Like sailing and rafting/tubing, it had a low number of base year trips and its supply and demand models were relatively weak. Backpacking, day hiking, and wildlife observation rank in the top fiveactivities for three of the four regions. However, there also are regional differences. Even though rafting/tubing is projected to have the largest percentage gap in the Rocky Mountain region, no other 
Table 20.-Projected gap between maximum preferred demand and expected supply of outdoor recreation trips away from home, measured as percentage difference by decade to 2040 , by region.

\begin{tabular}{lccccc} 
Resource category and & 1987 & & & \\
activity (national) & Trips $^{1}$ & 2000 & 2010 & 2020 & 2030 \\
region & $(\mathrm{mm})$ & $\mathrm{D} / \mathrm{S} / \mathrm{G}^{2}$ & $\mathrm{D} / \mathrm{S} / \mathrm{G}$ & $\mathrm{D} / \mathrm{S} / \mathrm{G}$ & $\mathrm{D} / \mathrm{S} / \mathrm{G}$ \\
\hline
\end{tabular}

Land

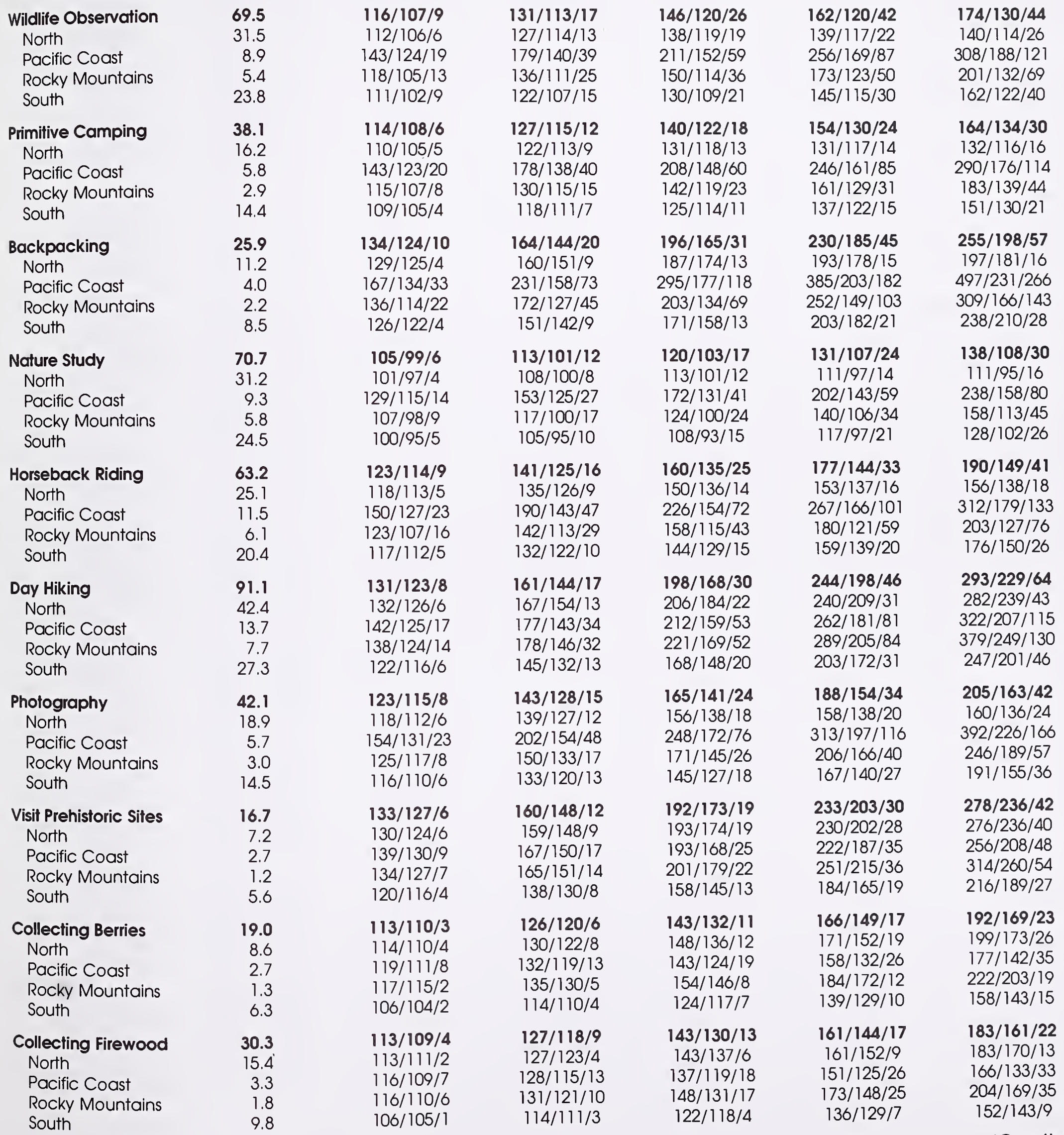

(Continued) 
Table 20.-(continued).

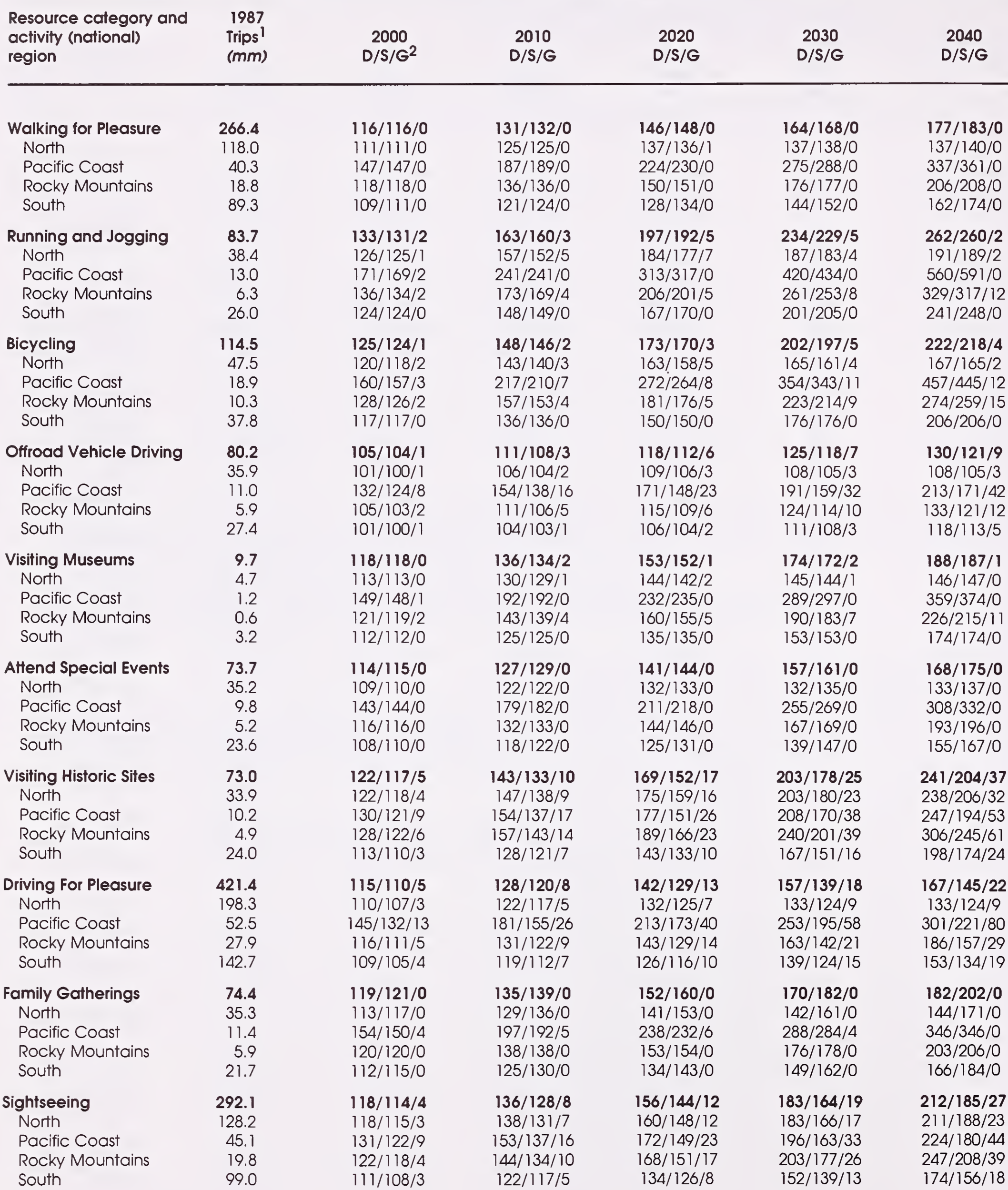

(Continued) 
Table 20.-(continued).

\begin{tabular}{|c|c|c|c|c|c|c|}
\hline $\begin{array}{l}\text { Resource category and } \\
\text { activity (national) } \\
\text { region }\end{array}$ & $\begin{array}{l}1987 \\
\text { Trips } 1 \\
(\mathrm{~mm})\end{array}$ & $\begin{array}{c}2000 \\
D / S / G^{2}\end{array}$ & $\begin{array}{l}2010 \\
D / S / G\end{array}$ & $\begin{array}{c}2020 \\
D / S / G\end{array}$ & $\begin{array}{c}2030 \\
D / S / G\end{array}$ & $\begin{array}{c}2040 \\
D / S / G\end{array}$ \\
\hline
\end{tabular}

$\begin{array}{lr}\text { Picnicking } & 262 . \\ \text { North } & 115.7 \\ \text { Pacific Coast } & 36.6 \\ \text { Rocky Mountains } & 20 . \\ \text { South } & 89.7 \\ & \\ \text { Developed Camping } & 60.5 \\ \text { North } & 26.8 \\ \text { Pacific Coast } & 8.5 \\ \text { Rocky Mountains } & 4.9 \\ \text { South } & 20 .\end{array}$

262.1
115.7
36.6
20.1
89.7
60.5
26.8
8.5
4.9
20.3

Water

Canoeing/kayaking
North
Pacific Coast
Rocky Mountains
South
Stream/Lake Swimming
North
Pacific Coast
Rocky Mountains
South
39.7
17.1
5.2
3.0
14.4

238.7

104.9

31.8

15.3

86.6

\section{Rafting/Tubing \\ North \\ Pacific Coast \\ Rocky Mountains \\ South}

Rowing/Paddling, etc.
North
Pacific Coast
Rocky Mountains
South
Motorboating
North
Pacific Coast
Rocky Mountains
South
Waterskiing
North
Pacific Coast
Rocky Mountains
South
Outdoor Pool Swimming
North
Pacific Coast
Rocky Mountains
South
Sailing
North
Pacific Coast
Rocky Mountains
South

$108 / 110 / 0$
$103 / 105 / 0$
$137 / 139 / 0$
$109 / 110 / 0$
$103 / 106 / 0$
$120 / 120 / 0$
$115 / 115 / 0$
$153 / 150 / 3$
$121 / 121 / 0$
$114 / 116 / 0$

$117 / 120 / 0$

$111 / 114 / 0$

$166 / 171 / 0$

$119 / 122 / 0$

$109 / 113 / 0$

$137 / 138 / 0$

$131 / 131 / 0$

$197 / 194 / 3$

$141 / 141 / 0$

$127 / 132 / 0$

$113 / 113 / 0$
$109 / 111 / 0$
$141 / 133 / 8$
$116 / 113 / 3$
$107 / 108 / 0$

$105 / 108 / 0$

$101 / 105 / 0$

$131 / 131 / 0$

$105 / 105 / 0$

$100 / 106 / 0$

$111 / 123 / 0$

$106 / 116 / 0$

$146 / 160 / 0$

$132 / 131 / 1$

$100 / 114 / 0$

$112 / 110 / 2$

$108 / 107 / 1$

$136 / 131 / 5$

$114 / 112 / 2$

107/106/1

$106 / 107 / 0$

$102 / 104 / 0$

$128 / 128 / 0$

$106 / 108 / 0$

$102 / 104 / 0$

$111 / 112 / 0$

$107 / 107 / 0$

$136 / 134 / 2$

$112 / 114 / 0$

$106 / 107 / 0$

$137 / 135 / 2$

$132 / 134 / 0$

$167 / 162 / 5$

$141 / 134 / 7$

$130 / 126 / 4$

$145 / 141 / 4$

$137 / 137 / 0$

$200 / 189 / 11$

$150 / 140 / 10$

$133 / 130 / 3$
$126 / 131 / 0$
$116 / 121 / 0$
$189 / 201 / 0$
$126 / 131 / 0$
$112 / 120 / 0$
$155 / 158 / 0$
$145 / 145 / 0$
$238 / 238 / 0$
$157 / 158 / 0$
$137 / 145 / 0$

$140 / 138 / 2$

$132 / 135 / 0$

$210 / 183 / 27$

$147 / 138 / 9$

$124 / 125 / 0$

$117 / 128 / 0$

$108 / 121 / 0$

$169 / 175 / 0$

$116 / 115 / 1$

$105 / 122 / 0$

$164 / 182 / 0$

$156 / 171 / 0$

$275 / 308 / 0$

$245 / 206 / 39$

$128 / 155 / 0$

$136 / 130 / 6$

$129 / 125 / 4$

$192 / 177 / 15$

$140 / 132 / 8$

$122 / 119 / 3$

$117 / 122 / 0$

$110 / 115 / 0$

$162 / 164 / 0$

$116 / 123 / 0$

$108 / 112 / 0$

$131 / 132 / 0$

$123 / 122 / 1$

$185 / 182 / 3$

$131 / 139 / 0$

$118 / 121 / 0$

$205 / 200 / 5$

$196 / 206 / 0$

$298 / 281 / 17$

$217 / 195 / 22$

$181 / 172 / 9$

$237 / 226 / 11$

$218 / 222 / 0$

$425 / 384 / 41$

$256 / 218 / 38$

$197 / 188 / 9$
$136 / 145 / 0$

$116 / 122 / 0$

$219 / 241 / 0$

$140 / 147 / 0$

$121 / 131 / 0$

$173 / 178 / 0$

$147 / 145 / 2$

$290 / 294 / 0$

$182 / 184 / 0$

$153 / 165 / 0$

$157 / 153 / 4$

$131 / 136 / 0$

$258 / 217 / 41$

$174 / 158 / 16$

$139 / 139 / 0$

$124 / 140 / 0$

$107 / 125 / 0$

$189 / 202 / 0$

$125 / 124 / 1$

$111 / 135 / 0$

$215 / 229 / 0$

$148 / 172 / 0$

$442 / 472 / 0$

$417 / 289 / 128$

$176 / 202 / 0$

$150 / 142 / 8$

$129 / 125 / 4$

$229 / 206 / 23$

$160 / 148 / 12$

$135 / 129 / 6$

$123 / 131 / 0$

$110 / 117 / 0$

$178 / 183 / 0$

$124 / 133 / 0$

$113 / 119 / 0$

$141 / 144 / 0$

$123 / 123 / 0$

$211 / 209 / 2$

$144 / 156 / 0$

$127 / 131 / 0$

$242 / 237 / 5$

$203 / 222 / 0$

$389 / 365 / 24$

$272 / 238 / 34$

$215 / 202 / 13$

$293 / 279 / 14$

$225 / 239 / 0$

$603 / 537 / 66$

$342 / 278 / 64$

$244 / 231 / 13$
$144 / 156 / 0$

$115 / 125 / 0$

$254 / 289 / 0$

$156 / 166 / 0$

$131 / 145 / 0$

$186 / 195 / 0$

$149 / 152 / 0$

$351 / 364 / 0$

$210 / 214 / 0$

$174 / 188 / 0$

$169 / 163 / 6$

$131 / 138 / 0$

$318 / 257 / 61$

$205 / 180 / 25$

$158 / 155 / 3$

$129 / 152 / 0$

$107 / 131 / 0$

$212 / 233 / 0$

$136 / 133 / 3$

$118 / 151 / 0$

$255 / 267 / 0$

$145 / 175 / 0$

$720 / 722 / 0$

$724 / 406 / 318$

$249 / 265 / 0$

$159 / 150 / 9$

$129 / 125 / 4$

$273 / 240 / 33$

$184 / 166 / 18$

$150 / 142 / 8$

$127 / 138 / 0$

$110 / 120 / 0$

$196 / 205 / 0$

$132 / 145 / 0$

$118 / 127 / 0$

$148 / 152 / 0$

$124 / 125 / 0$

$241 / 240 / 1$

$159 / 176 / 0$

$137 / 142 / 0$

$269 / 267 / 2$

$208 / 240 / 0$

$502 / 470 / 32$

$337 / 287 / 50$

$255 / 236 / 19$

$335 / 322 / 13$

$232 / 256 / 0$

$844 / 743 / 101$

$453 / 351 / 102$

$302 / 282 / 20$

(Continued) 


\begin{tabular}{|c|c|c|c|c|c|c|}
\hline $\begin{array}{l}\text { Resource cafegory and } \\
\text { activity (national) } \\
\text { region }\end{array}$ & $\begin{array}{l}1987 \\
\text { Trips }^{1} \\
(\mathrm{~mm})\end{array}$ & $\begin{array}{c}2000 \\
D / S / G^{2}\end{array}$ & $\begin{array}{c}2010 \\
D / S / G\end{array}$ & $\begin{array}{c}2020 \\
D / S / G\end{array}$ & $\begin{array}{c}2030 \\
D / S / G\end{array}$ & $\begin{array}{c}2040 \\
D / S / G\end{array}$ \\
\hline \multicolumn{7}{|l|}{ Snow and Ice } \\
\hline $\begin{array}{l}\text { Cross-Country Skiing } \\
\text { North } \\
\text { Pacific Coast } \\
\text { Rocky Mountains } \\
\text { South }\end{array}$ & $\begin{array}{l}9.7 \\
5.3 \\
1.3 \\
1.2 \\
2.0\end{array}$ & $\begin{array}{c}147 / 125 / 22 \\
140 / 112 / 28 \\
198 / 154 / 44 \\
157 / 132 / 25 \\
135 / 126 / 9\end{array}$ & $\begin{array}{l}177 / 136 / 41 \\
174 / 120 / 54 \\
288 / 191 / 97 \\
206 / 155 / 51 \\
160 / 143 / 17\end{array}$ & $\begin{array}{c}199 / 142 / 57 \\
190 / 116 / 74 \\
364 / 211 / 153 \\
232 / 161 / 71 \\
164 / 146 / 18\end{array}$ & $\begin{array}{c}212 / 141 / 71 \\
154 / 88 / 66 \\
484 / 245 / 239 \\
284 / 181 / 103 \\
181 / 157 / 24\end{array}$ & $\begin{array}{c}195 / 126 / 69 \\
121 / 66 / 55 \\
615 / 277 / 338 \\
336 / 197 / 139 \\
194 / 165 / 29\end{array}$ \\
\hline $\begin{array}{l}\text { Downhill Skiing } \\
\text { North } \\
\text { Pacific Coast } \\
\text { Rocky Mountains } \\
\text { South }\end{array}$ & $\begin{array}{r}64.3 \\
32.7 \\
11.2 \\
8.9 \\
11.5\end{array}$ & $\begin{array}{l}153 / 160 / 0 \\
164 / 174 / 0 \\
173 / 178 / 0 \\
168 / 168 / 0 \\
142 / 136 / 6\end{array}$ & $\begin{array}{l}197 / 207 / 0 \\
229 / 243 / 0 \\
234 / 241 / 0 \\
233 / 230 / 3 \\
176 / 163 / 13\end{array}$ & $\begin{array}{c}247 / 256 / 0 \\
294 / 309 / 0 \\
288 / 295 / 0 \\
298 / 290 / 8 \\
202 / 184 / 18\end{array}$ & $\begin{array}{c}298 / 305 / 0 \\
317 / 336 / 0 \\
361 / 364 / 0 \\
398 / 376 / 22 \\
241 / 213 / 23\end{array}$ & $\begin{array}{c}333 / 338 / 0 \\
336 / 357 / 0 \\
441 / 436 / 5 \\
521 / 478 / 43 \\
284 / 243 / 41\end{array}$ \\
\hline
\end{tabular}

\footnotetext{
'Regional trips may not sum exactly to the national total because of rounding.

${ }^{2} D$ is the maximum preferred demand; $S$ is the expected supply; and $G$ is the percentage difference (gap) between demand and supply. D, S, and $G$ are all expressed as percentages of the 1987 base number of trips. In the projection base year of 1987, demand is assumed to equal supply with zero gap, that is, demand and supply are in equilibrium.

Source: Outdoor Recreation and Wilderness Assessment Research, USDA Forest Service, Athens, GA.
}

regions are projected tohaveany gap for that activity. While all regions are projected to have some gap for both visiting historic and prehistoric sites, only in the North region are these gaps among the top five activities with gaps.

Table 21.-Oufdoor recreation activities with the largest projected percentage gaps in 2040 , by region.

\begin{tabular}{|c|c|c|c|c|}
\hline \multirow[b]{2}{*}{ Activity } & \multicolumn{4}{|c|}{ Region } \\
\hline & $\begin{array}{c}\text { Pacific } \\
\text { North }\end{array}$ & $\begin{array}{l}\text { Rocky } \\
\text { Coosi }\end{array}$ & Mountains & South \\
\hline & \multicolumn{4}{|c|}{ Gap in percent (top five ranked gaps) } \\
\hline Cross-country skiing & $55(1)$ & $338(1)$ & $139(3)$ & $29(4)$ \\
\hline Rafting/tubing & - & - & $318(1)$ & - \\
\hline Day hiking & $43(2)$ & 115 & $130(4)$ & $46(1)$ \\
\hline Backpacking & 16 & $266(2)$ & $143(2)$ & $28(5)$ \\
\hline Wildlife observation & $26(5)$ & $121(5)$ & 69 & $40(2)$ \\
\hline Visiting prehistoric sites & $40(3)$ & 48 & 54 & 27 \\
\hline Photography & 24 & $166(3)$ & 57 & $36(3)$ \\
\hline Visiting historic sites & $32(4)$ & 53 & 61 & 24 \\
\hline Horseback riding & 18 & $133(4)$ & 76 & 26 \\
\hline Sailing & - & 101 & $102(5)$ & 20 \\
\hline
\end{tabular}

Source: Outdoor Recreation and Wilderness Assessment Research, USDA Forest Service, Athens, GA.
Regional differences are less apparent when examining projected gaps in terms of the absolute number of trips (table 22). Gaps in numbers of trips for sightseeing, driving for pleasure, and day hiking are among the top four activities with gaps in each of the four regions. Although no gap is projected for outdoor pool swimming for the North region, in each of the other regions that activity is projected to have relatively large gaps. In the two eastern regions, relatively large gaps in trips are predicted for wildlife observation, but not for horseback riding. The reverse is true for the two western regions.

Almost every activity for which a small or zero gap was projected occurs primarily in an environment with developed recreation facilities. Recent trends in developed land and water resources indicate that the supply of such recreation opportunities will continue to increase fast enough to absorb additional trip demands. Growth in recreation resources and facilities implies reduced cost for trip production. Lower trip costs induce households to produce more trips. As a result, supply and consumption is greater, and gaps are less likely.

Activities such as backpacking, day hiking, and wildlife observation that occur most often in less developed and more remote settings are projected, to a large extent, 
to experience growth in preferred demand that will outpace consumption and production of trips. The models predict that, as accessible recreation resources decline or fail to keep pace with growth in demand, households will not be able to produce the number of trips they would prefer to take in the future at the 1987 price. Here, preferred demand will exceed the expected supply of trips.

\section{CONCLUSIONS}

Changes in EROS values are determined by both projected population growth and expected changes in recreation resources. Resource trends for undeveloped recreation resources reflect broad social and land use trends, especially the conversion of land from open space to urbanized areas. Offsetting increases in undeveloped lands owned by state and federal agencies are not expected; therefore, opportunities for most undeveloped environments are expected to decline. Developed opportunities are expected to increase, because of both additional parkland acquisition by local governments, and increased opportunities provided by the private sector.

Much regional variation is masked by national level analyses. Each region has a unique combination of resources, travel behavior, population characteristics, and projected changes. In general, the western regions have greater amounts of resources, longer effective travel distances, and higher projected rates of population growth. Because of these combinations, EROS values are not expected to be as sensitive to population

Table 22.-Outdoor recreation activities with the largest projected gaps in absolute number of trips in 2040 , by region.

\begin{tabular}{lcccc}
\hline & \multicolumn{4}{c}{ Region } \\
\cline { 2 - 5 } Activity & $\begin{array}{c}\text { Pacific } \\
\text { North }\end{array}$ & $\begin{array}{c}\text { Rocky } \\
\text { Coast }\end{array}$ & Mountains & South \\
\hline & \multicolumn{4}{c}{} \\
& Gap in percent (top five ranked gaps) \\
Sightseeing & $29.5(1)$ & $19.8(2)$ & $7.7(4)$ & $17.8(2)$ \\
Driving for pleasure & $17.8(3)$ & $42.0(1)$ & $8.1(3)$ & $27.1(1)$ \\
Day hiking & $18.2(2)$ & $15.8(3)$ & $10.1(1)$ & $12.6(4)$ \\
Outdoor pool swimming & 0 & $11.8(5)$ & $10.0(2)$ & $14.2(3)$ \\
Visiting historic sites & $10.8(4)$ & 5.4 & 3.0 & 5.8 \\
Horseback riding & 4.5 & $15.3(4)$ & $4.6(5)$ & 5.3 \\
Wildlife observation & $8.2(5)$ & 10.8 & 3.7 & $9.5(5)$ \\
& \multicolumn{4}{c}{} \\
\hline
\end{tabular}

Source: Outdoor Recreation and Wilderness Assessment Research, USDA Forest Service, Athens, GA. increases as in the eastern regions. Although population is expected to grow more rapidly in the West, longer effective travel distances mean that the demand for recreation resources will be spread over a broader area. In the eastern regions, where travel distances are shorter, resources closer to population centers will bear a relatively greater share of increased demand. As the capacity of these resources is reached more rapidly, the availability of these resources for other recreating households will be reduced. Therefore, a higher level of use pressure can be expected to be put on recreation resources located near population centers in the eastern half of the country, compared to resources in the West.

In general, the rate of demand growth is expected to be greatest for some strenuous activities, including running and jogging, backpacking, bicycling, hiking, swimming and cross-country skiing. Many of these activities take place in undeveloped environments and on or near trails. Other activities with generally high rates of projected demand growth use scenic or historic resources, including visiting historic and prehistoric sites, sightseeing, and photography. Many of these activities also are those expected to have the greatest absolute number of trips demanded over the next 50 years.

Gap analyses represent the combined results of expected shifts in demand based on trends in population characteristics and income changes, as well as expected trends in resource availability and trip substitution. Gaps partly depend on projected changes in recreation resources, because consumption of recreation trips depends on the availability of effective recreation opportunities. This implies that a large gap for an activity can be reduced by increasing recreation resources that support the activity.

Projected growth in the expected supply of trips is generally highest for activities that occur in developed recreation environments, because those are the opportunities that are predicted to increase most in availability. Projected consumption increases in these activities are further bolstered by the anticipated decline in substitute, less developed, opportunities. As less developed opportunities become more scarce or equivalently, more expensive, households are expected to substitute trips to less expensive developed environments for more expensive undeveloped environments. As a result, these types of activities are generally expected to have gaps at or near zero.

Examining the activities that are projected to have the largest gaps indicates that across all regions, a high degree of use pressure will be placed on trail and scenic 
resources near population centers. These are the resources that provide a primary resource base for activities, such as day hiking, backpacking, horseback riding, sightseeing, and pleasure driving. Because much wildlife observation activity also occurs on or near trails, this activity's gaps are also affected. Even though the western regions may have substantial amounts of trail resources, many are not located near cities. For all regions, resources near population centers are most important in providing effective recreation opportunities. These also are the resources that will undergo the most land conversion and use pressures as the United States population continues to grow.

Some regional differences are evident from the gap analyses as well. In the western regions, some waterbased recreation activities will have large gaps, including swimming and rafting/tubing. This may indicate increased pressure by the public for additional waterbased recreation resources. In the East, opportunities for wildlife observation or green space activities may be insufficient to meet recreation demand, especially opportunities near population centers. An important part of the resource base in this part of the country that has in the past supported such activities are privately owned rural lands.

In general, regional demand and supply analyses appear to be a significant improvement over national analyses. Further research is needed to improve the capacity of these types of analyses to provide guidance for assessing aggregate future trends. These research areas include:

1. Tracking region-specific trends in resource changes, rather than applying national trends to regional resource levels, to increase the specificity of effectiveness and resource values;

2. Estimating region-specific demand and consumption models, to allow coefficients to vary across regions;

3. Improving methods for estimating EROS values, for example by eliminating the simplifying assumption of linearly declining effectiveness decay weights;

4. Examining substitution between activities for recreating households, in order to improve the substitution measure in consumption models;
5. Examining the stability of coefficients in demand and consumption models over time, to see if projections for these values can be accomplished with static coefficients;

6. Building explanatory models using the individual approach, as opposed to the zonal method;

7. Incorporating a measure of ethnicity into the models, especially in light of increasing ethnic diversity.

These types of additional research can yield another round of improvements in the methods and results for assessing the demand and supply of outdoor recreation in the United States.

\section{LITERATURE CITED}

Becker, G. S. 1965. A theory of the allocation of time. Economic Journal. 75:493-517.

Bockstael, N. E.; McConnell, K. E. 1981. Theory and estimation of the household production function for wildlife recreation. Journal of Environmental Economics and Management. 8:199-214.

Clawson, M. 1984. Effective acreage for outdoor recreation. Resources. 78:1-7.

Cordell, H. K.; Bergstrom, J. C. 1989. Theory and techniques for assessing the demand and supply of outdoor recreation in the United States. Res. Pap. SE-275. Athens, GA: U.S. Department of Agriculture, Southeastern Forest Experiment Station. 33 p.

Cordell, H. K.; Bergstrom, J. C. 1991. A methodology for assessing national outdoor recreation demand and supply trends. Leisure Sciences. 13:1-20.

Cordell, H. K.; Bergstrom, J. C.; Hartmann, L. A.; English, D. B. K. 1990. An analysis of the outdoor recreation and wilderness situation in the United States: 1989-2040. Gen. Tech. Rep. RM-189. Fort Collins, CO: U.S. Department of Agriculture, Rocky Mountain Forestand RangeExperimentStation.113 p.

Cordell, H. K.; English, D. B. K. 1985. Recreational travel distances to identify supply inventory radii.In: Anderson, L.M., ed. Proceedings of the 1984 Southeastern Recreation Research Conference; 1984 February 1617; Asheville, NC. Athens, GA: University of Georgia, Institute for Behavioral Research. 
Cordell, H. K.; English, D. B. K.; Bergstrom, J. C. 1989. Measuring and projecting effective recreation opportunities in the United States. In: Proceedings of Benchmark 1988: A national forum on outdoor recreation in the United States. Gen. Tech. Rep SE-52. Asheville, NC: Southeastern Forest Experiment Station. 498p.

English, D. B. K.; Cordell, H. K. 1993. Effective Recreation Opportunity Set (EROS) Index: A computable recreation supply measure. Res. Pap. SE-286. Asheville, NC:U.S. Department of Agriculture, Southeastern Forest Experiment Station. $11 \mathrm{p}$.

Fesenmaier, D. R.; Leiber, S. R. 1987. Spatial structure and behavior response in outdoor recreation participation. Geografiska Annaler. 67B:131-138.

Flather, C. H.; Hoekstra, T. W. 1989. An analysis of the wildlife and fish situation in the United States: 19892040. Gen. Tech. Rep. RM-178. Fort Collins, CO: U.S. Department of Agriculture, Rocky Mountain Forest and Range Experiment Station. 147 p.
Harrington, W. 1987. Measuring Recreation Supply. Washington, DC: Resources for the Future. $77 \mathrm{p}$.

Kim, S.; Fesenmaier, D. R. 1990. Evaluating spatial structure effects in recreation travel. Leisure Sciences. 12:367-381.

McConnell, K. E. 1975. Some problems in estimating the demand for outdoor recreation. American Journal of Agricultural Economics. 57:330-334.

United Ski Industries Association. [n.d.] Ski Statistics Fact Sheet. 3p. Available from: United Ski Industries Association, 8377-B Greensboro Drive, McLean, VA 22102-3587. [Written in 1991.]

United States Department of Commerce, Bureau of Economic Analysis. 1991. BEA regional projections to 2040. Volume 1: States.

United States Department of the Interior, National Park Service. 1986. 1982-1983 nationwide recreation survey. Washington, DC: U.S. Government Printing Office. $93 \mathrm{p}$. 



\begin{abstract}
English, Donald B. K.; Betz, Carter, J.; Young, J. Mark; Bergstrom, John C.; Cordell, H. Ken. 1993. Regional demand and supply projections for outdoor recreation. Gen. Tech. Rep. RM-230. Fort Collins, CO:U.S. Department of Agriculture, Forest Service, Rocky Mountain Forest and Range Experiment Station. 39 p.

This paper develops regional recreation supply and demand projections, by combining coefficients from the national 1989 RPA Assessment models with regional regressor values. Regional recreation opportunity estimates also are developed, based on regional travel behavior. Results show important regional variations in projections of recreation opportunities, trip supply, and trip demand.
\end{abstract}

Keywords: Outdoor recreation, regional demand, regional supply, recreation projections, demand/supply comparisons, projection models, trends.

USDA policy prohibits discrimination because of race, color, national origin, sex, age, religion, or handicapping condition. Any person who believes he or she has been discriminated against in any USDA-related activity should immediately contact the Secretary of Agriculture, Washington, DC 20250. 



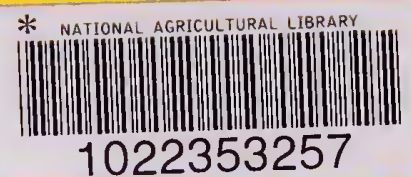

